\title{
Using Brexit to Identify the Nature of Price Rigidities
}

\author{
Bart Hobijn \\ Arizona State University \\ CRIW \\ Fernanda Nechio \\ Federal Reserve Bank of San Francisco \\ Adam Hale Shapiro \\ Federal Reserve Bank of San Francisco
}

April 2019

Working Paper 2019-13

https://www.frbsf.org/economic-research/publications/working-papers/2019/13/

\section{Suggested citation:}

Hobijn, Bart, Fernanda Nechio, Adam Hale Shapiro. 2019. "Using Brexit to Identify the Nature of Price Rigidities,” Federal Reserve Bank of San Francisco Working Paper 201913. https://doi.org/10.24148/wp2019-13

The views in this paper are solely the responsibility of the authors and should not be interpreted as reflecting the views of the Federal Reserve Bank of San Francisco or the Board of Governors of the Federal Reserve System. 


\title{
Using Brexit to Identify the Nature of Price Rigidities*
}

\author{
Bart Hobijn \\ Arizona State University \\ CRIW
}

Fernanda Nechio

FRB San Francisco

Adam Hale Shapiro

FRB San Francisco

April 30, 2019

\begin{abstract}
Using price quote data that underpin the official U.K. consumer price index (CPI), we analyze the effects of the unexpected passing of the Brexit referendum to the dynamics of price adjustments. The sizable depreciation of the British pound that immediately followed Brexit works as a quasi-experiment, enabling us to study the transmission of a large common marginal cost shock to inflation as well as the distribution of prices within granular product categories. A large portion of the inflationary effect is attributable to the size of price adjustments, implying that a time-dependent price-setting model can match the response of aggregate inflation reasonably well. The state-dependent model fares better in capturing the endogenous selection of price changes at the lower end of the price distribution, however, it misses on the magnitude of the adjustment conditional on selection.
\end{abstract}

JEL classification codes: E31, D40, F31.

Keywords: inflation, price dynamics, micro data, menu cost, time-dependent pricing, statedependent pricing.

*The views expressed in this paper are those of the authors and do not necessarily reflect the position of the Federal Reserve Bank of San Francisco or the Federal Reserve System. We are grateful to Neil Gerstein and Kevin Vo for their excellent research assistance. 


\section{Introduction}

On June 23, 2016 a small majority of 51.9 percent of voters in Great Britain voted in favor of a British exit, "Brexit," from the European Union. The outcome of this referendum was a major surprise. Opinion polls, political prediction markets, as well as foreign exchange futures all signaled a broad consensus, even on the morning of the vote, that the "remain" vote would prevail (The Economist, 2016). The referendum results astonished the public, rocked careers of politicians in the "remain" camp, and, most importantly for this paper, shocked the foreign exchange market. The result was a sharp depreciation of the British pound (GBP).

In the aftermath of the referendum the trade-weighted effective exchange rate of the GBP depreciated by more than 10 percent. Even by the end of 2016, the Pound had depreciated by more than 6 percent against the currencies of Britain's major trading partners. Because the U.K. is a very open economy, with imports amounting to about 30 percent of its GDP, such a negative terms of trade shock increases the cost of many products in the U.K. that are either imported or produced using imported intermediates. The Brexit shock propagated through the distribution of prices for different items in the British Consumer Price Index (CPI) and resulted in an acceleration of inflation after June 2016.

In this paper we use the Brexit shock to study the dynamics of price adjustments. The Brexit shock works as a quasi-experiment that enables us to study the transmission of a large common shock to marginal costs through the distribution of prices and inflation. This allows us to distinguish between different types of price setting models with nominal rigidities.

We uncover two key features of the response of prices to the Brexit shock. First, the shock induces an increase in log prices which is mostly driven by an increase in the magnitude of price adjustments rather than the incidence of price changes. Second, price dispersion does not change very much. This is so because, even though the variance of inflation increases, there is a sizable mean reversion of prices. This mean reversion is largely driven by the selection in which prices change in that the incidence of price changes increases more for lower prices than for higher prices. The result is that lower prices increase faster than higher ones.

Our paper is part of a long tradition of analyzing exchange rate pass-through on inflation, e.g., Gagnon (2009), Gopinath and Itskhoki (2010) Auer and Schoenle (2016), Auer, Burstein and Lein (2017), among others. Our analysis differs from these studies in two dimensions.

First, we use an explicit quasi-experimental setup in which we split the British CPI microdata sample up into "treatment" and "control" groups. The "treatment" group includes items whose cost is directly affected by the unanticipated movement in the exchange rate because they are either imported or their production relies on imported intermediates. The "control" group 
includes goods and services that are domestically produced with low import content. We then use local projections (Jordà, 2005) to estimate the impact of the shock through its differential effect on the dynamics of the distribution of log prices of the "treatment" compared to the "control" group.

Second, this dynamic differences-in-differences (DID) approach allows us to explicitly focus on the effects of the shock on the distribution of log prices over time and relate our estimates to the transitional dynamics of commonly-used models of price setting under nominal rigidities.

Thus, our analysis adds to the extensive literature that uses micro data on prices to document certain empirical properties of the distribution of inflation rates and map them into canonical macroeconomic models of price adjustment. ${ }^{1}$ We make two contributions to this literature.

The first is that we use data on the level of the log prices in the micro data in addition to the information on the change of the log prices, i.e., the inflation rate, that is used in the studies above. The reason we can use these price level data is because we construct a panel of separate log-price distributions within the elementary item categories. Specifically, these items are classified in very detailed categories at the regional level by type of shop over timewhat we refer to as a "bin." 2 Thus, the products within each of these bins are very similar and this makes differences in their price levels directly interpretable. We use the cross-sectional dimension, across the log-price distributions within a bin for a given month, for our construction of the "treatment" and "control" groups in our quasi-experimental setup.

Our second contribution to this strand of the literature is that we consider a larger set of empirical properties of the distribution of log prices than previous studies have done. In this respect, we build on Berger and Vavra (2017) and Berger and Vavra (2018), who study the variance of inflation rates on top of the, most commonly analyzed, mean of the inflation rate that is driven by the incidence and magnitude of price changes. We use a new decomposition of the first- and second-order moments of the distribution of log prices that nests the existing analyses of both the mean and the variance of inflation rates. This decomposition contains several, never before analyzed, components that exploit the information on the relationship between the level of log prices and the incidence and magnitude of price changes. These new components turn out to be readily interpretable in that they can be used to directly distinguish between different types of models of price setting under nominal rigidities.

\footnotetext{
${ }^{1}$ For example, Bils and Klenow (2004), Dhyne et al. (2006), Klenow and Malin (2010), and Nakamura and Steinsson (2008), Nakamura and Steinsson (2010), Midrigan (2011), Kehoe and Midrigan (2015), and Alvarez, Lippi and Passadore (2016). In particular, for the U.K. Kryvtsov and Vincent (2014) and Dixon and Tian (2017).

${ }^{2}$ An example of such a bin would be marmalade at supermarket chains in the Southwest of England.
} 
The tight link between the empirical components of the distribution of log prices in our decomposition and canonical theoretical models of price setting under nominal rigidities allows us to use the Brexit shock to identify important aspects of the nature of price rigidities in the data. Our second key empirical finding, namely that of a mean reversion in price setting because lower prices change more frequently in response to the shock, is direct evidence of state-dependent price setting by British firms. Previous studies (Alvarez, Lippi and Passadore, 2016; Klenow and Kryvtsov, 2008) pointed out that, under small shocks, time-dependent and state-dependent price setting models are observationally equivalent. However, our results seem to reject the time-dependent models. This means that, in the context of these papers, the Brexit shock, which amounts to an average 4 percent cost increase for the items in our "treatment" group, is a large shock.

However, it turns out that the off-the-shelf state-dependent menu-cost model, as used in Golosov and Lucas (2007) for example, misses on the magnitude of the adjustment conditional on selection. In the state-dependent model, the narrowing of the price adjustment bands implies that prices at the higher end of the distribution change by larger amounts, which is inconsistent with the data. The result is that the menu-cost model implies a counterfactually rapid adjustment of the log-price level.

Even though a basic time-dependent model, based on Calvo (1983), cannot match our second key empirical finding of state-dependent price setting, it outperforms the canonical menu-cost model in terms of matching the speed of the dynamics of the adjustment of the log price level. This largely reflects our first key empirical finding that these dynamics are mostly driven by the changes in the magnitude of the price changes rather than the incidence. Hence, what is most important is to have a model that does well in terms of matching the magnitude of price changes rather than the incidence.

The bottom line is that the time-dependent model better captures the response of the magnitude of price changes, and hence, it better matches the dynamic response of inflation to the Brexit shock than the canonical menu-cost model. The latter model, however, better captures the endogeneity of the decision to change prices but does not match the magnitude of price changes conditional on changing prices.

\section{U.K. economic performance and the Brexit referendum}

To understand the macroeconomic context within which we analyze the transmission of the exchange rate shock associated with Brexit, in this section we look at the exchange rate, economic activity, and inflation in Great Britain before and after the referendum. We then focus, 
in more detail, on the increase in inflation in the U.K. following the Brexit vote and show that it is concentrated in goods and services with a large import content.

Figure 1 shows three main macroeconomic indicators for the U.K. economy, namely; the exchange rate, real GDP growth, and CPI inflation. To put the macroeconomic conditions during the Brexit vote in context it is worthwhile to consider the 15 years leading up to it.

From 2001 through 2007 the British economy performed well. The exchange rate was relatively stable, real GDP growth came in at an average annualized rate of 2.9 percent, and inflation hovered between 1 and 3 percent.

Because of the global financial crisis of 2008 and the ensuing euro crisis in 2011, the British economy had seen turbulent times in the eight years preceding the Brexit referendum. British banks were hit hard by the financial crisis, including the bankruptcy of Northern Rock, and the financial crisis resulted in a 30 percent depreciation of the exchange rate and a 6 percent decline in real GDP. The depreciation of the GBP, combined with a spike in global commodity and food prices, caused an initial spike in inflation to 5 percent.

However, a subsequent cut in Value Added Tax (VAT) rates and the economic slack during the recession resulted in CPI inflation retreating to 1 percent. A reversal of the VAT cuts of 2009 and an additional VAT hike in 2011 as an "emergency budget" measure led to another spike in inflation in 2011. After that, inflation declined. Just like in many other industrialized countries, largely driven by declining energy prices in 2014 and 2015, inflation dropped well below 1 percent in the two years preceding the Brexit vote. As can be seen from Figure 1, in the 12 months leading up to the Brexit referendum, CPI inflation had only been 0.3 percent in the United Kingdom, real GDP growth had been 2 percent, and GBP had depreciated in light of diverging monetary policy expectations in the United Kingdom and the United States.

Then the surprise outcome of the Brexit referendum happened in June 2016. As can be seen from Figure 1a, immediately following the referendum, the GBP depreciated more than 10 percent against the currencies of the U.K.'s main trading partners. This depreciation of the GBP was a surprise to the extent that the outcome of the Brexit referendum was a surprise. Economic forecasts had predicted a substantial depreciation of the GBP in case the "Yes" vote would prevail (International Monetary Fund, 2016), but the referendum outcome surprised the public, market participants and policymakers (The Economist, 2016).

Figure 2 more clearly illustrates the effect of the surprising referendum result by plotting the daily value of the GBP against the dollar and the euro. The GBP sharply depreciated against both currencies on the day of the Brexit referendum.

Forecasts of the short-run effect of a "Yes" vote on economic activity varied substantially. Some forecasters predicted a recession in the U.K., while others foresaw only a slight slowdown in 
GDP growth. The International Monetary Fund (IMF), for example, entertained two scenarios; an adverse and a limited scenario (International Monetary Fund, 2016). The former had the U.K. slip into a recession after the Brexit "Yes" vote in 2017, resulting in about a five percent in decline in the level of GDP compared to the baseline. The latter scenario included a small decline in real GDP growth in the U.K. after a "Yes" vote, resulting in a 1.5 percent decline in the level of GDP compared to the baseline.

In hindsight, as can be seen from Figure 1b, the IMF's limited impact scenario turned out to be relatively accurate. In the 18 months after the Brexit referendum, 4-quarter GDP growth in the U.K. decelerated from 2 percent to 1.2 percent. Of course, it is hard to know what the counterfactual would have been. However, over the same period, GDP growth in other G-7 economies, accelerated rather than decelerated. Thus, in the wake of the Brexit referendum, the British economy has underperformed compared to other economies. Born et al. (2017) estimate this underperformance to add up to 1 percent of GDP. ${ }^{3}$

Most importantly for this paper is that, even though the Brexit vote has induced some slowdown in the growth of economic activity in the U.K., it has not resulted in a major downturn or recession. That is, the immediate effects on the real side of the British economy have been limited, and in line with the IMF's limited scenario.

What has also come in line with the IMF's limited scenario has been the effects of Brexit on British CPI inflation. Because about a fifth of the expenditures in the British CPI is on directly imported goods and services or on high-import content goods and services due to imported intermediates, the 10 percent surprise depreciation of the pound resulted in a substantial increase in the cost of final goods and services sold to consumers. ${ }^{4}$ This resulted in a run-up of inflation from 0.7 percent in the year before the referendum to 3.1 percent by November 2017 (Figure 1c).

The post-Brexit acceleration of inflation was due to an increase in the inflation rate of tradables goods and services. Figure 3 shows this by plotting the time series of the overall CPI inflation rate from Figure 1c together with the inflation rates of the two-digit Classification of Individual Consumption According to Purpose (COICOP) tradables and nontradables categories as classified by Allington, Kattuman and Waldmann (2005). Figure 3 reveals that the inflation rate of nontradables did not change much after the Brexit referendum, and what increased was the inflation rate of tradable goods and services. ${ }^{5}$ We rely on this different re-

\footnotetext{
${ }^{3}$ Estimates of long-run effects of Brexit on the level of U.K. GDP range from this 1 percent, based on static trade effects (Reenen, 2016), to even larger than 10 percent (International Monetary Fund, 2016), depending on what one assumes about the structure of post-Brexit trade relationships between the U.K. and European Union

${ }^{4}$ The 2014 Analytical Input-Output tables published by the Office for National Statistics (ONS) estimate the import content of British domestic consumption to be 19.4 percent. See Table 1 for details.

${ }^{5}$ Other studies have noted that goods and services with a high import content have seen higher inflation
} 
sponse of prices of tradables and nontradable goods for our identification strategy to uncover the nature of price rigidities in the U.K.

\section{Quasi-experimental setup using British CPI micro-data}

The above observation that the increase in British inflation after the Brexit referendum was solely driven by tradables and that the inflation rate of nontradables barely responded, it the basis for the quasi-experimental setup that we use in the rest of this paper.

Our main approach is to split up the sample of goods and services covered in the microdata with price quotes underlying the British CPI published by the ONS into "treatment" and "control" groups. These data have been used in previous research by Kryvtsov and Vincent (2014) and Dixon and Tian (2017), among others. ${ }^{6}$ We then study the differential impact of the Brexit shock on the distributions of log prices in these two groups.

The "treatment" group consists of a set of goods and services that are part of the tradable COICOPs plotted in Figure 3. These are goods and services whose cost of production increased due to the depreciation of the GBP and that drove the increase in aggregate inflation in the U.K. plotted in the figure.

The "control" group consists of a sample of goods and services that are domestically produced using few or no imported intermediates. These are part of the nontradable COICOPs in Figure 3 and their inflation rate was hardly affected by the unanticipated passing of the Brexit referendum.

To understand how we categorize goods and services in these "treatment" and "control" groups, it is useful to consider an example of how a particular price quote is classified in the micro-data we use. Figure 4 illustrates how a "price quote of a jar of Marmalade at a Tesco supermarket in Southampton" is classified.

The ONS collects price quotes for goods and services grouped at the elementary item level. The jar of marmalade is part of the elementary item "Jar of jam, 340-454g." These jars of jam are themselves part of the 3-digit COICOP group 01.1.8 "Sugar, jam, honey, chocolate, and confectionary." This group is part of the 2-digit COICOP classification 1.1 "Food" in Table 1.

Price quotes for the British CPI are collected in 12 regions across the United Kingdom. Southampton is in the Southeast region. Some price quotes, such as the ones from online stores, are not particularly associated with an outlet in a specific region and are, instead,

after Brexit: “. . for each 10 percentage point rise in a product group's import share, inflation increased by 0.71 percentage points in the year after the vote" (Breinlich et al., 2017).

${ }^{6} \mathrm{~A}$ comparison of the aggregated price quote data with the published CPI measure is available in Appendix B.2. 
classified as "nationwide."

The outlets where the price quotes are collected are split into two shop types; independent outlets, and shops that are part of a chain with multiple outlets. Each specific outlet is assigned a shop code as its unique identifier. That is, in our example from Figure 4, Tesco is a supermarket chain with multiple outlets, and the particular Tesco in Southampton where the price quote was collected has the unique, fictitious, shop code of 123 .

We use the sampling structure explained above and depicted in Figure 4 to construct a panel of distributions of prices of elementary items by region and shop type. We refer to each unique combination of these three variables, (item, region, shop type), as a bin. For each month, we construct the distribution of log prices for all price quotes in each bin. The result is that we have data on a panel of distributions of log prices, each distribution associated with a particular bin in our data. ${ }^{7}$

It is these bins that we group into our "treatment" and "control" samples. We do so based on the degree of tradability of the item, e.g., the jar of marmalade in our example above, covered in the bin. We consider two sources of information to assess the degree of tradability of goods and services. The first is are the 2014 Analytical Input-Output tables for the U.K. published by the ONS. These tables provide the import content of goods and services based on the U.K. input-output tables The second source is Allington, Kattuman and Waldmann (2005), that we also used in, Figure 3. They provide a binary classification of COICOPs into tradables versus nontradables. Table 1 summarizes the findings of both sources. The import content column of Table 1 shows the fraction of the cost of the expenditures of the different COICOPs that can be traced back to imports. The second column reports Allington, Kattuman and Waldmann (2005)'s classification.

Note from Table 1 how, at the 2-digit level of aggregation, the tradability classification and import shares are closely aligned. This 2-digit level of aggregation is the lowest level that both these measures have in common. Because we exploit differences in the inflationary effect of the Brexit shock across COICOPs at a lower levels of aggregation in the rest of this paper, we use a combination of both the tradability classification from Allington, Kattuman and Waldmann (2005) at lower levels of aggregation, and the import content shares at the level of aggregation reported in the table.

With this in mind, we split our cross-section of bins up into treatment, control, and ambiguous groups. We do so by jointly evaluating two criteria related to the elementary item in each bin. The first is whether the low-level COICOP group that the item with which the bin is associated is classified as tradable or nontradable (as reported in the last column of Table 1).

\footnotetext{
${ }^{7}$ For more detailed information on how bins were constructed please see Appendix B.1.
} 
The second is the import-content tertile of the 2-digit COICOP group, from Table 1, that the item belongs to. The joint evaluation of these two criteria allows us to split our sample of bins up into a 2-by-3 grid, which we report in Table $2 .^{8}$

As our treatment sample we use price quotes for tradable items with a high import content. This sample consists of the bins in the lower-right cell of Table 2. These are the bins for elementary items that are both classified as tradable by Allington, Kattuman and Waldmann (2005) and are in the top tertile of import content shares in Table 1. This covers 529 bins with region-shop-type-specific distributions of prices for 40 different elementary items.

As our control sample, we use bins associated with items that are classified as nontradable and are in the bottom tertile in terms of the import share. This sample consists of bins in the upper-left cell of Table 2 and covers 938 bins with distributions of prices for 80 different elementary items.

Thus, we choose the treatment and control samples to include the items whose costs are the most and the least affected by imports, respectively. We do so to maximize the difference in the impact of the surprise depreciation of the GBP after Brexit on the distributions of prices in both samples. We exclude the bins in the other cells of Table 2 for which the importance of imports for the costs of the items is more ambiguous. ${ }^{9}$

Of course, for any quasi-experimental setup one needs to not only define the treatment and control samples, but also the outcomes one considers to measure the impact of the treatment. Our samples consist of distributions of log prices by bin. So, the outcomes we consider are the differences in the dynamics of the distributions of log prices of the treatment and control samples after the Brexit referendum. In the next section, we introduce a new decomposition of these dynamics, the components of which we use as our outcome measures in the rest of our analysis.

\section{Components of dynamics of the distribution of log prices}

One of the contributions of our analysis is that we use a new decomposition of the changes in the distribution of $\log$ prices in a bin in our data to assess the impact of Brexit on prices

\footnotetext{
${ }^{8}$ The price quotes we include are "regular" prices and exclude sales. We do so because regular prices are best captured by the dynamics of price-setting models with nominal rigidities (Kehoe and Midrigan, 2015). Moreover, we also exclude petrol (due to its high price volatility) and clothing because, as Liegey (1993) pointed out, many matched price quotes are not for identical items but for close substitutes. Results do not substantially change when these categories are not excluded in the analysis. See Appendix B.3 for full details of how the data were cleaned.

${ }^{9}$ The specific numbers and estimates that we present in the rest of our analysis do, of course, depend on this split of our sample. However, the qualitative conclusions from our analysis do not change when we alter our sample split shown in Table 2.
} 
in the U.K. This decomposition is general and can be applied to other micro-data sets. In addition, there are many aspects of the distribution of prices that one can track over time. Our decomposition, in particular, applies to the change in the average log price and the change in the variance of log prices following the Brexit shock. The reason we this decomposition is that it splits the changes in these first- and second-order moments of the distribution of log prices in components whose dynamics are distinct under different price setting models.

Because most theoretical models of price setting under nominal rigidities abstract from entry and exit of items in the market, the decomposition described below, as well as our empirical analysis in Section 5, relies on matched price quotes. These are price quotes for which we do observe the price both in two consecutive months. ${ }^{10}$ Other empirical studies of CPI micro-data also have focused on a matched sample (e.g., Bils and Klenow, 2004; Gagnon, 2009; Dixon and Tian, 2017).

We denote the number of price quotes in a bin in month $t$ by $n_{t}$ and index these price quotes by $j=1 \ldots n_{t}$. We denote the $\log$ of the price $j$ in month $t$ as $p_{j, t}$. For each of these matched observations we can approximate the percent change in the price, $\pi_{j, t}$, by the change in the log of the price level, i.e., $\pi_{j, t}=p_{j, t}-p_{j, t-1}$.

We consider the sample mean and variance of these matched log prices and write them, respectively, as:

$$
\bar{p}_{t}=\frac{1}{n_{t}} \sum_{j=1}^{n_{t}} p_{j, t} \text { and } \sigma_{t}^{2}=\frac{1}{n_{t}} \sum_{j=1}^{n_{t}}\left(p_{j, t}-\bar{p}_{t}\right)^{2} .
$$

Our focus in this paper is on the change in these two sample moments of the distribution of $\log$ prices between months $t-1$ and $t$, i.e., on $\Delta \bar{p}_{t}=\bar{p}_{t}-\bar{p}_{t-1}$ and $\Delta \sigma_{t}^{2}=\sigma_{t}^{2}-\sigma_{t-1}^{2}$.

We focus on $\Delta \bar{p}_{t}=\bar{p}_{t}-\bar{p}_{t-1}$ because the inflation rate for an item in our sample (in the $\mathrm{CPI}), \pi_{t}$, is measured using an equally-weighted geometric price index over all matched price quotes. That is, the item-level inflation rate in the CPI is the sample mean of the percent change in prices for all matched observations, i.e.,

$$
\pi_{t}=\frac{1}{n_{t}} \sum_{j \in M} \pi_{j, t}=\Delta \bar{p}_{t}
$$

which equals the change in the mean of log prices of the matched price quotes. Thus, we focus on the mean of the log price levels by bin for our analysis.

\footnotetext{
${ }^{10}$ We present our decomposition here for matched price quotes and derive a generalized decomposition that takes into account entry and exit in Appendix A, where we denote the set of these matched price quotes by $M$. In practice, most entry and exit of price quotes in the CPI micro-data has little to do with economic concepts of entry and exit. Instead, it largely reflects seasonal item rotations in stores and sample rotation of outlets and items. In our data, some exit is due to ONS price collectors not being able to find the same item in the store in two consecutive months.
} 
We also consider $\Delta \sigma_{t}^{2}=\sigma_{t}^{2}-\sigma_{t-1}^{2}$ because the degree of price dispersion in many models with nominal rigidities is directly related to the welfare losses due to nominal rigidities (Eusepi, Hobijn and Tambalotti, 2011; Nakamura et al., 2016). Moreover, considering the dynamics of this second moment helps us distinguish between several models of price setting under nominal rigidities in the data.

We split the matched observations up into two groups. The first are those price quotes for which the price stays the same between $t-1$ and $t$. These $n_{t}^{S}$ observations, which we denote by the set $S$, are those, $j$, for which $p_{j, t-1}=p_{j, t}$. The second set, which we denote by $C$, consists of those $n_{t}^{C}$ price quotes for which the price changes, i.e., $p_{j, t-1} \neq p_{j, t}$. Note that $n_{t}=n_{t}^{S}+n_{t}^{C}$.

The fraction of matched items for which the price changes over a given month $t$, i.e., $\delta_{t}=\frac{n_{t}^{C}}{n_{t}}$, is known as the incidence of price changes. ${ }^{11}$ The size of the price changes, i.e., $\Delta \bar{p}_{t}^{C}$, is the magnitude of price changes. The variance of $\log$ prices, i.e., $\sigma_{t}^{2}$, is a measure of price dispersion. The variance of inflation rates for the items whose price change, i.e., $\left(\sigma_{\pi, t}^{C}\right)^{2}$, is a measure of inflation dispersion. Our decomposition also encompasses a covariance term, $\sigma_{\pi, p_{t-1}, t-1}^{C}$, that allows us to quantify the degree of mean reversion in log prices in the data. To see how this covariance term implies a mean reversion, note that the ratio $\frac{\sigma_{\pi, p_{t-1}, t-1}^{C}}{\left(\sigma_{t-1}^{C}\right)^{2}}$ is the regression coefficient for a regression of the percent change in prices that change $\left(\Delta \bar{p}_{t}^{C}\right)$ on the initial log prices $\left(p_{t-1}\right)$. In the case in which all prices in $C$ are set to the same reset price, then there is perfect mean reversion in prices that change, and this coefficient is equal to -1 .

These unconditional and conditional first- and second-order moments are either measures that are commonly analyzed in empirical studies of price rigidities or are directly related to theoretical concepts from and implications of models of price setting under nominal rigidities. ${ }^{12}$

\subsection{Decomposition of the inflation rate}

Splitting the sample of matched price quotes up into these two subsets allows us to write inflation as the product of the incidence and magnitude of price change, i.e.,

$$
\pi_{t}=\Delta \bar{p}_{t}=\delta_{t} \Delta \bar{p}_{t}^{C}
$$

\footnotetext{
${ }^{11}$ This incidence of price changes is the focus of many studies of nominal rigidities (Bils and Klenow, 2004; Nakamura and Steinsson, 2010; Dhyne et al., 2006, for example), including for the United Kingdom (Dixon and Tian, 2017).

${ }^{12}$ Our decomposition divides $\Delta \bar{p}_{t}$ and $\Delta \sigma_{t}^{2}$ into conditional moments are listed in the Appendix Table A1. The note below the table provides examples of how these moments are defined. Before we use them in our decomposition, it is useful to first consider the economic interpretation of some of them.
} 
This allows us to use a shift-share-type decomposition to analyze the variation in the inflation rate in the bin over time.

Equation (4) rewrites the previous one to yield an additive decomposition that allows us to both analyze cumulative inflation, i.e., the log change in the price index since a base period, as well as the variance of inflation. It shows that inflation can be high, relative to its average, for three reasons. First, because the magnitude of price changes is high. Second, because the incidence of price changes is high and many items change prices. Third, because of the positive interaction between times when many prices change, and times when prices change by a lot. These three reasons are, respectively, captured in the second through fourth terms on the right-hand side of:

$$
\pi_{t}=\bar{\delta} \bar{\pi}^{C}+\underbrace{\bar{\delta}\left(\pi_{t}^{C}-\bar{\pi}^{C}\right)}_{\text {Magnitude }}+\underbrace{\bar{\pi}^{C}\left(\delta_{t}-\bar{\delta}\right)}_{\text {Incidence }}+\underbrace{\left(\delta_{t}-\bar{\delta}\right)\left(\pi_{t}^{C}-\bar{\pi}^{C}\right)}_{\text {Interaction }}
$$

where $\bar{\pi}$ and $\bar{\delta}$ are, respectively, average inflation and average incidence over the sample period $t=1 \ldots T$, such that, $\bar{\pi}^{C}=\frac{1}{T} \sum_{1}^{T} \pi_{t}^{C}$ and $\bar{\delta}=\frac{1}{T} \sum_{1}^{T} \delta_{t}$.

Cumulative inflation can be built and split into the same parts as $\pi_{t}$ in equation (4) by summing both the left- and right-hand sides over the relevant time period. We use this decomposition of cumulative inflation in our empirical analysis.

\subsection{Decomposition of the change in price dispersion}

In Appendix A, we show that the change in the dispersion of the matched price quotes in a month can be split up into four parts:

$$
\begin{aligned}
\Delta \sigma_{t}^{2} & =\overbrace{\sigma_{\pi, t}^{2}}^{\text {Variance of inflation }}+\overbrace{\begin{array}{c}
\text { Variance of } \\
\text { magnitude }
\end{array}}^{\delta_{t}\left(\sigma_{\pi, t}^{C}\right)^{2}}+\underbrace{\left(1-\delta_{t}\right) \delta_{t}\left(\pi_{t}^{C}\right)^{2}}_{\text {Variance of incidence }}+\underbrace{2 \delta_{t} \sigma_{\pi, p_{t-1}, t}^{C}}_{\begin{array}{c}
\text { Mean reversion of } \\
\text { magnitude }
\end{array}}+\underbrace{2 \delta_{t} \pi_{t}^{C}\left(\bar{p}_{t-1}^{C}-\bar{p}_{t-1}\right)}_{\text {Mean reversion of incidence }}
\end{aligned}
$$

The first line in this equation shows that the increase in the variance of log prices is due to the dispersion in inflation rates, i.e., $\sigma_{\pi, t}^{2}$, plus the extent to which these price changes revert to the mean, i.e., $\sigma_{\pi, p_{t-1}, t}$. The latter term is best understood by realizing that if high prices tend to increase faster than low ones, then this causes the spread in the distribution of log prices to increase (and vice versa). The higher the variance and the less the mean reversion (i.e., the more the price expansion) will act to increase the dispersion of prices. 
The variance and mean reversion terms can be further decomposed into "magnitude" and "incidence" components. The first term on the second line captures the the variance of magnitude of the changes in the price quotes in $C$. The variance of inflation will be smaller the more similar the magnitudes of price changes. The second term captures the variance of incidence of price changes. Whether or not a matched price quote changes between months $t-1$ and $t$ can be interpreted as a draw from a Bernoulli distribution with mean $\delta_{t}$. The associated variance is, hence, $\delta_{t}\left(1-\delta_{t}\right)$. This term will be maximized when half of the prices in the distribution adjust. The next two terms are decompositions of the mean reversion of inflation. The term, $\delta_{t} \sigma_{\pi, p_{t-1}, t}^{C}$, reflects the degree to which inflation reduces (or magnifies) initial differences in log prices among the prices that change. We call this the mean reversion of magnitude of price changes. If prices at the bottom the distribution increase by a larger amount than prices at the top of the distribution then there will be more mean reversion (i.e., less price expansion). The last term reflects the extent to which the fact that the prices that change are not randomly selected from the matched price quotes reduces (or magnifies) price differences. We call this the mean reversion of incidence of price changes. If prices are more apt to adjust from the bottom of the distribution than the top of the distribution than mean reversion will increase (and dispersion will expand less).

We are not the first study to use CPI micro-data to split up inflation into parts due to the incidence and magnitude of price changes (e.g., Bils and Klenow, 2004, Dhyne et al., 2006, Gagnon, 2009, and Dixon and Tian, 2017). Previous analyses of price dispersion have also focused on specific components of equation (5). ${ }^{13}$ However, we are the first study to decompose the changes in price dispersion in a comprehensive fully additive fashion. This full decomposition allows us to quantify which components drive the response of price dispersion to a large shock, which can then be compared to different price-setting models.

What enables us to calculate this full decomposition is that we can use the information on price levels necessary to calculate the terms related to the mean reversion of inflation in (5). This is because we consider the distributions for very specific items, in particular regions, sold at specific types of stores, which makes price quotes in each bin comparable to each other.

\footnotetext{
${ }^{13}$ Most studies have analyzed the dispersion of price changes, i.e., the variance of the inflation rates, $\sigma_{\pi, t}^{2}$ (e.g., Bils and Klenow, 2004, Gagnon, 2009, Klenow and Kryvtsov, 2008, Berger and Vavra, 2017, and Berger and Vavra, 2018). However, many studies in the industrial organization literature, e.g., Cornia, Gerardi and Shapiro (2012), have examined the dispersion of price levels.
} 


\section{$5 \quad$ Key empirical findings}

In this section we use a dynamic DID method, based on Jordà (2005), to estimate the difference in the impact of the unexpected passing of the Brexit referendum on the distributions of log prices of our treatment and control samples. This yields two main findings. First, the Brexit shock is followed by an increase in log prices, which is mostly driven by the size of price adjustments rather than the frequency (incidence) of price changes. Second, price dispersion does not change significantly. This is so because although the variance of inflation increases, there is a sizable mean reversion of prices, i.e., lower prices increase faster than high ones, which offsets the former increase. The second-moment decomposition shows that the data features endogenous selection of the incidence of price adjustments, which help explain the small (dynamic) response of the variance of log prices.

\section{Summary statistics before and after Brexit referendum}

Before we turn to our regression analysis, we first compare our treatment (Treat.) with our control sample (Cont.) as well as with the whole sample (All) before the Brexit shock occurred in Table 3. The table provides summary statistics for three periods, pre-Brexit, post-Brexit and a longer benchmark sample that allows for a comparison of price statistics further away from the shock.

The pre- and post-Brexit years together are the period we consider in our empirical analysis in this section. The benchmark period was chosen based on the fact that the GBP effective exchange rate, GDP growth, and CPI inflation volatility were relatively stable over this time frame (see Figure 1). We use this period for calibration of our models in the next section.

The top half of Table 3 reports the contributions of the magnitude of price changes, $\pi_{t}^{C}$, and the incidence of price changes, $\delta_{t}$, to the log of price changes. ${ }^{14}$ Similarly, the bottom half of the table reports the contributions of the variance, $\sigma_{\pi, t}^{2}$, and mean reversion, $2 \sigma_{\pi, p_{t-1}, t}$, of inflation to the change in dispersion of prices, based on (5). ${ }^{15}$ In addition, the table contains the variance of log prices as well as the number of price quotes used to calculate the statistics reported.

Note that for almost all combinations of periods and measures, the point estimates for the whole sample (all) is in between those for the treatment (treat.) and control (cont.) samples. This is because the whole sample also includes the items classified as ambiguous. Note that for

\footnotetext{
${ }^{14}$ The constributions are calculated by applying a variance decomposition to (3), i.e., $\operatorname{Var}\left[\pi_{t}\right]=$ $\operatorname{Cov}\left[\pi_{t}, \bar{\pi}^{C}\left(\delta_{t}-\bar{\delta}\right)\right]+\operatorname{Cov}\left[\pi_{t}, \bar{\delta}\left(\pi_{t}^{C}-\bar{\pi}^{C}\right)\right]+\operatorname{Cov}\left[\pi_{t},\left(\delta_{t}-\bar{\delta}\right)\left(\pi_{t}^{C}-\bar{\pi}^{C}\right)\right]$.

${ }^{15}$ In the Appendix Table A3, we expand Table 3 to include all subcomponents from these decompositions (as described in equation (3) and the second line of equation (5)).
} 
all the three subsamples and periods the mean reversion of inflation is negative, indicating that higher prices exhibit lower inflation than lower prices across the whole sample in our data.

When we look across the three subperiods, the control sample shows relatively similar magnitudes of the contributions of the subcomponents of (3) and (5). This is not the case for the treatment sample. For the treatment sample the contributions of the components of (3) and (5) during the benchmark and pre-shock periods are of comparable magnitude. However, in the post-shock period the magnitude of price changes, i.e., $\Delta \bar{p}_{t}^{C}$, the variance of inflation, i.e., $\sigma_{\pi, t}$, and the mean reversion of inflation, i.e., $2 \sigma_{\pi, p_{t-1}, t}$, are all much larger than in the other two periods reported. This is indicative of the significant effect of Brexit on the price setting in the treatment sample that we quantify using our dynamic DID below.

When we compare the treatment with the control sample, we find significant differences between them in the benchmark and pre-shock periods. This is reflective of the quasi-experimental nature of our analysis. If we would have been able to assign the treatment randomly across items in the British CPI then we could have made sure that these two samples looked the same in terms of the summary statistics in Table 3 during the benchmark and pre-shock periods. However, this is not feasible in our setup. To make sure these differences are not driving our key results, we account for these types of pre-treatment differences between the treatment and control items in a robustness check that uses a synthetic-control approach at the end of this section.

\section{Dynamic DID specification using local projection method}

We apply the local projection method of Jordà (2005) to the change in the mean of log prices, $\Delta \bar{p}_{t}$, and the change in price dispersion, $\Delta \sigma_{t}$, as well as their components from (3) and (5) respectively. This method allows us to estimate the differential impact of the Brexit shock on these variables between the treatment and control samples over different horizons.

The Brexit shock is captured by the dummy variable $\mathbb{B}_{t}$ that equals one from June 2016 onwards. We differentiate between the treatment and control samples using the dummy variable $\mathbb{T}_{i}$ which is one if item $i$ is classified as tradable, as in Table 2, and is part of our treatment sample. The horizon is the number of months over which we capture the impact of the shock. We denote it by $h \in\{0, \ldots, 12\}$ and study the response of the cumulative change in the firstand second-order moments of the distribution of log prices, and their components, over the year following the Brexit referendum. 
We quantify this response using the regression equation:

$$
y_{i, t+h}-y_{i, t-1}=\gamma_{i}+\delta^{h} \mathbb{B}_{t}+\beta^{h} \mathbb{B}_{t} * \mathbb{T}_{i}+\sum_{c=1}^{C} \sum_{k=1}^{12}\left(\alpha_{c, k}^{h} \Delta C_{c, i, t-k}+\phi_{c, k}^{h} \Delta C_{c, i, t-k} * \mathbb{T}_{i}\right)+\varepsilon_{i, t} .
$$

We estimate equation (6) for two sets of dependent variables $y_{i, t}:{ }^{16}$

(i) the log price level, $p_{i, t}$, and its three time-varying components described in equation (4),

(ii) the variance of $\log$ prices, $\sigma_{i, t}^{2}$, and its components described in equation (5).

The coefficients of interest are the estimates of $\beta^{h}$, which trace out the path of $y_{i, t+h}$ for the treatment group items relative to control group items in the $h$ months following Brexit. Under the null hypothesis, the coefficients $\beta^{h}$ all equal zero, meaning that the treatment and control groups remained on the same relative pre-Brexit path during the post-Brexit period. Therefore, our identification assumption attributes any deviation of $\beta^{h}$ away from zero as being caused by the exchange rate shock. Note that since our analysis relies on matched (by shop) price quotes between months $t-1$ and $t$, the dependent variable for each horizon $h$ is measured as the sum of monthly changes for each type of variable $y_{i, t+h}$, providing the cumulative effect of the shock on variable $y_{i, t+h}$ by time $t+h .{ }^{17}$

We include a set of control variables, $C_{c, i, t}$, aimed to remove pre-Brexit trends. In particular, we include 12 lags of the time-varying components of equations (4) and (5), depending on the dependent variable being used. That is, $C=3$ in case (i) when we consider components of equation (4), and $C=4$ in case (ii) when $y_{i, t}$ is one of the components of equation (5). We also include 12 lags of each component interacted with the treatment-group dummy, which allows for different pre-trends for treatment- and control-group items. To account for Nickell bias we employ the Arellano-Bond estimator. ${ }^{18}$ We cluster standard errors at the 64 lowest (three-digit) levels of aggregation in the COICOP classification.

Although all measures are constructed at the bin level, regressions are run at the more aggregated elementary-item level to reduce attenuation bias, such that $i$ in the regression indicates an elementary item. To do so, we aggregate the bin measures up to the elementary-item

\footnotetext{
${ }^{16}$ See Appendix $\mathrm{C}$ for detailed regression specifications. In that we also consider a third set of dependent variables given by the $10^{t h}, 50^{t h}$ and $90^{t h}$ percentiles of the log price level. Results are reported in the Appendix Figure A3.

${ }^{17}$ For example, for the log price level we have $p_{i, t+h}-p_{i, t-1}=\sum_{\tau=1}^{h} \pi_{i, t+\tau}$. The inflation rate for item $i$ is $\pi_{i, t}=\sum \omega_{b, i} \pi_{b, i, t}$, where $\omega_{b, i}$ is the bin weight and $\pi_{b, i, t}=\sum \omega_{s, b, i}\left(p_{s, b, i, t}-p_{s, b, i, t-1}\right)$ is the matched-shop $\log$ change in prices for item $i$ in $b$ and shop $s$, and $\omega_{s, b, i}$ are shop weights provided by ONS.

${ }^{18}$ Results did not substantially change using OLS or replacing the Arellano-Bond instruments with their principal components (Bai and Ng, 2010).
} 
level using bin-specific weights provided by ONS. ${ }^{19}$

The regression is estimated for the sample period from June 2015 to June 2017, and we take the first year of the sample (June 2015 to May 2016) as the pre-Brexit period, and the second year of the sample (July 2016 to June 2017) as the post-shock period. The results obtained using this regression specification are plotted in Figures 5 and 6 .

\section{Impact of Brexit on price-setting decisions for tradable CPI items}

The solid black line in the top panel of Figure 5 shows the estimated $\beta^{h}$ for regressions in which the variable $y_{i, t+h}$ corresponds to the mean log price level. In the bottom three panels, the figure reports the estimated $\beta^{h}$ for regressions in which the variable $y_{i, t+h}$ corresponds to the three time varying components of the change in log prices; i.e., magnitude, incidence and interaction. Because we include the same explanatory variables in all regressions underlying the panels in Figure 5, the estimated $\beta^{h}$ 's in the bottom three panels add up to the one reported in the top panel.

The top panel shows the accumulated effect of the Brexit shock on the mean log prices of tradable CPI items (relative to nontradables). It reveals a significant, but delayed, impact that peaks about 8 months after the referendum. The decomposition in the bottom panels shows our first key finding. The bulk (about 75 percent) of the increase in log prices of tradables after the Brexit shock is associated with an increase in the magnitude of price adjustments, while the incidence of price changes rises by a stastically-significant but economically small amount.

The solid line in the top panel of Figure 6 shows the estimated $\beta^{h}$ for regressions in which the variable $y_{i, t+h}$ corresponds to the variance in log prices. Our second key finding can be seen from the middle and bottom panels of the figure. They report the estimated $\beta^{h}$ for regressions in which the variable $y_{i, t+h}$ corresponds to the components of the change in the variance of $\log$ prices as described in equation (5). The top panel shows a very limited effect of Brexit on price dispersion. The middle panels explain this finding by showing that, while there is an increase in the variance of inflation, this effect is more than offset by the covariance term, i.e., the mean reversion in prices. The negative and relatively sizable mean reversion indicates that it is lower prices that increased by more in response to the shock, compressing the distribution of log prices. The net effect, if anything, is a small decline in price dispersion rather than an increase (as seen on the top panel). The bottom panels complement this analysis by reporting the remaining components of the decomposition described in equation (5). It shows that the

\footnotetext{
${ }^{19}$ For example, the inflation rate for item $i$ is $\pi_{i, t}=\sum \omega_{b, i} \pi_{b, i, t}$, where $\omega_{b, i}$ is the weight for bin $b$ and item $i$. Bin weights are provided by ONS which refers to them as "stratum" weights. See Appendix B.1 for more details on bin construction and Figure 4 for an illustrative example of the data structure.
} 
mean reversion of incidence more than offsets the small but positive effects of the increases in the variance of magnitude and incidence.

Note that the finding that the variance of magnitude increases is in line with the results of Berger and Vavra (2017), who also find that, in the U.S. data, $\sigma_{\pi, t}^{C}$ increases in response to exchange rate shocks. Of course, our decomposition implies that this only partially captures the impact of the exchange rate on the disparity of price levels. By focusing only on $\sigma_{\pi, t}^{C}$ in equation (5), one might conclude that price disparities increase when the disparity of inflation rates increases. However, the addition of the terms that involve the price levels, most importantly, the covariance term $\sigma_{\pi, p_{t-1}, t}^{C}$, reveals that that is not the case. The increase in the variance of inflation rates of prices that change, i.e., of $\sigma_{\pi, t}^{C}$, in response to the shock, is more than offset by the mean-reversion term.

\section{Robustness and validation}

One possible concern about our empirical findings is that there is substantial heterogeneity between goods and services even at the bin level. This heterogeneity would be reflected in terms of disparities in $(\log )$ prices that are not necessarily related to differences in markups or marginal costs but instead to quality differences across goods. This is the reason that researchers have mostly ignored the micro-data on price levels. However, if this heterogeneity was of firstorder importance in the data, one would not expect movements in the covariance between inflation and the initial price level to be such an important component of price dynamics, as Figure 6 shows, because this heterogeneity would drive the term related to this covariance in the decomposition to zero.

Another source of concern regarding our empirical findings and methodology may arise from the intrinsic differences between the tradable and nontradable sectors. In an ideal experiment, the treated and control units are identical, on average, in the absence of the treatment. However, the summary statistics, reported in Tables 3, indicate some differences in the treatment and control items in the nontreatment periods (i.e., the benchmark and pre-Brexit periods). Most notably, the treated group (tradables) includes items whose prices change more frequently than those in the control group. Although the local projection method used in this study includes controls aimed to correct for pre-Brexit trends, it does not account for differential effects of the treatment in the post-Brexit period. For example, flexibly-priced items may react to a cost shock differently than sticky-priced items do. Such differences in characteristics of the treated group from the comparison group could potentially convolute the empirical results.

To alleviate the latter concern, we apply the synthetic control method of Abadie, Diamond 
and Hainmueller (2010) to our data. More specifically, we construct a "synthetic" control group as a convex combination of control items that most closely resembles the characteristics of the treated group. The synthetic control group is build such that it matches the frequency of price changes $\left(\delta_{t}\right)$, the inflation of price changes $\left(\pi_{t}^{C}\right)$, the variance of inflation $\left(\sigma_{\pi, t}^{2}\right)$, and the mean reversion of price changes $\left(2 \sigma_{\pi, p_{t-1}, t}\right)$ of the treated group over the pre-Brexit period (2015m62016m5). The synthetic control matches the statistics of the treatment group quite well (see Tables A4 and A5). We then perform our empirical approach to obtain the relative response of the treated group to the Brexit shock.

The results from the synthetic control method, reported in Appendix Section C.1, reinforce our main empirical findings and show that the effect of the exchange rate shock on tradables through local projections and the synthetic control methods are quite close to one another across all variables.

\section{Comparison with canonical models of price rigidities}

To understand what the estimated impulse responses teach us about the nature of nominal rigidities underlying the British CPI, we compare the empirical impulse responses with those obtained from two canonical models of price setting. They are the basic model of price setting with time-dependent nominal rigidities, based on Calvo (1983), and a model with statedependent price setting under menu costs, as in Golosov and Lucas (2007) for example. Our analysis is definitely not the first to compare these two models. ${ }^{20}$ What is new here is that we study the differences between these two models in terms of their transitional dynamics of the components of price setting dynamics, from equations(4) and (5), in response to a permanent cost shock. We compare these transitional dynamics with the estimates from the previous section.

In both models, we consider an economy composed of two sectors, $i=1,2$ that differ in the extent to which the exchange rate affects the marginal cost of production through imported intermediates. We index these sectors by $i$ to emphasize that they are the equivalent of an elementary item in our data. We interpret the sector $i=1$, where the marginal cost does not depend on imported intermediates, as part of our control sample. The other sector, $i=2$, is part of the treatment sample in the lower-right cell of the same table. Just like in our empirical analysis, we study the difference in the responses of these two sectors to a change in the cost of imported intermediates, induced by a surprise change in the nominal exchange rate.

\footnotetext{
${ }^{20}$ Most of the existing studies focus on differences in steady-state properties of these two models under various rates of steady-state inflation (e.g., Alvarez, Lippi and Passadore, 2016; Nakamura et al., 2016).
} 


\subsection{Model with nominal rigidities and permanent cost shock}

Both sectors are made up of a continuum of monopolistic competitors of mass one that produce varieties. We index these producers by $j \in(0,1)$ and interpret the prices they set as the theoretical counterparts of the price quotes we measure in the data. The inputs used in the production distinguish the two types of output, $i=1,2$. The first type, $i=1$, is produced solely using labor, while the second, $i=2$, is produced using both labor and imported intermediates. In both sectors, producers of different varieties have different total factor productivity (TFP) levels, which we denote by $A_{i, j, t}$.

Variety $j$ in sector 1 is produced using a linear production technology in labor:

$$
Y_{1, j, t}=A_{1, j, t} L_{1, j, t}
$$

Variety $j$ in sector 2 is produced using a Cobb-Douglas technology in labor and imported intermediates:

$$
Y_{2, j, t}=A_{2, j, t} \tilde{A} L_{2, j, t}^{1-\alpha} M_{j, t}^{\alpha} \text {, where } \tilde{A}=\left[\left(\frac{1-\alpha}{\alpha}\right)^{\alpha}+\left(\frac{\alpha}{1-\alpha}\right)^{1-\alpha}\right]
$$

In both sectors, producers of each variety can flexibly adjust their input choices. All producers take the prices of the inputs as given. In particular, $W_{t}$ is the nominal wage and $P_{t}^{*}$ is the price of imported intermediates denominated in GBP. Because of constant returns to scale for each producer, the average cost equals marginal cost, which are given by:

$$
M C_{i, j, t}=\left\{\begin{array}{l}
\frac{W_{t}}{A_{i, j, t}} \text { if } i=1 \\
\frac{1}{A_{i, j, t}} W_{t}^{1-\alpha}\left(P_{t}^{*}\right)^{\alpha} \text { if } i=2
\end{array} .\right.
$$

Note that the marginal cost of production in sector 2 is directly impacted by the price of imported intermediates, which, in turn, is directly affected by changes in the exchange rate.

Producers of varieties in each of these two markets face Constant Elasticity of Substitution (CES) demand functions, elasticity of substitution between these varieties $\varepsilon$, given by:

$$
Y_{i, j, t}=\left(\frac{P_{i, j, t}}{P_{i, t}}\right)^{-\varepsilon} Y_{i, t}, \text { where } P_{i, t}=\left[\int_{0}^{1} P_{i, j, t}^{1-\varepsilon} d i\right]^{\frac{1}{1-\varepsilon}}
$$

Demand for each of the goods is determined by their price, $P_{i, t}$, relative to the aggregate price level, $P_{t}$, and the level of aggregate demand, $Y_{t}$. The sector-specific demand functions are given 
by:

$$
Y_{i, t}=\left[\int_{0}^{1} Y_{i, j, t}^{\frac{\varepsilon-1}{\varepsilon}} d j\right]^{\frac{\varepsilon}{\varepsilon-1}}=\left(\frac{P_{i, t}}{P_{t}}\right)^{-\theta} Y_{t}, \text { where } i=1,2 \text { and } 0<\theta<\varepsilon
$$

To simplify notation in the rest of our exposition, we introduce $\mathcal{P}_{i, j, t}=\frac{P_{i, j, t}}{P_{i, t}}, \mathcal{P}_{i, t}=\frac{P_{i, t}}{P_{t}}$, and $\mathcal{P}_{t}^{*}=\frac{P_{t}^{*}}{P_{t}}$ for the relevant relative prices, as well as $\mathcal{W}_{t}=\frac{W_{t}}{P_{t}}$ for the real wage. This notation, along with the cost and demand functions above, allows us to write the real profits of varieties' producers as

$$
\Pi_{i}\left(\mathcal{P}_{i, j, t} ; A_{i, j, t}, \mathcal{P}_{i, t}, \mathcal{S}_{t}\right)=\left\{\begin{array}{l}
Y_{t}\left(\mathcal{P}_{i, t}^{-\theta} \mathcal{P}_{i, j, t}^{-\varepsilon}\right)\left(\mathcal{P}_{i, t} \mathcal{P}_{i, j, t}-\frac{1}{A_{i, j, t}} \mathcal{W}_{t}\right) \text { if } i=1 \\
Y_{t}\left(\mathcal{P}_{i, t}^{-\theta} \mathcal{P}_{i, j, t}^{-\varepsilon}\right)\left(\mathcal{P}_{i, t} \mathcal{P}_{i, j, t}-\frac{1}{A_{i, j, t}}\left(\mathcal{W}_{t}\right)^{1-\alpha}\left(\mathcal{P}_{t}^{*}\right)^{\alpha}\right) \text { if } i=2
\end{array}\right.
$$

where $\mathcal{S}_{t}=\left\{\mathcal{W}_{t}, \mathcal{P}_{t}^{*}, P_{t} Y_{t}\right\}$ are the aggregate variables that firms take as given when they make their price-setting decisions.

\section{Evolution of $A_{i, j, t}, \mathcal{P}_{i, t}$, and $\mathcal{S}_{t}$}

The log of the firm-specific productivity level, $a_{i, j, t}$, evolves independently across firms over time according to an $\mathrm{AR}(1)$-process of the form

$$
a_{i, j, t}=\rho a_{i, j, t-1}+u_{i, j, t}, \text { where } u_{i, j, t} \sim N\left(0, \sigma_{u}\right) .
$$

We introduce this firm-specific productivity shock to match the substantial level of price disparities we find during the benchmark period, reported in the bottom row of Table 3.

The relative price level of sector $i, \mathcal{P}_{i, t}$, is an equilibrium variable that is determined by the price-setting choices of all the firms in sector $i$. Hence, we solve for a fixed point in which the price-setting response of firms to the path of $\mathcal{P}_{i, t}$ aggregates to.

To keep our analysis focused on the first-order effect of the change in $\mathcal{P}_{t}^{*}$ on the distribution of prices in the two sectors, we abstract from general equilibrium effects of the shock to $\mathcal{P}_{t}^{*}$ on the aggregate variables, $P_{t}, Y_{t}, \mathcal{W}_{t}$, and $\mathcal{P}_{t}^{*}$.

Similar to Hobijn, Ravenna and Tambalotti (2006), we assume that relevant aggregate variables are constant along the equilibrium path. In particular, we assume that aggregate inflation is constant at the target rate, i.e., $\pi_{t}=\frac{P_{t+1}}{P_{t}}-1=\bar{\pi}$. The real interest rate is constant such that $r_{t}=r$. In addition, we abstract from growth in overall activity such that output, $Y_{t}$, and the real wage, $\mathcal{W}_{t}$, are constant over time. ${ }^{21}$

\footnotetext{
${ }^{21}$ We abstract from growth, because adding growth to the model only complicates the math and does not materially change the results.
} 
Most importantly, we assume that there is a surprise increase in the relative price of imported intermediates at time $t$. Consistent with our assumptions about other aggregate variables, $\mathcal{P}_{t}^{*}$ is constant along the equilibrium path except that it jumps at time $t=0$, i.e.,

$$
\mathcal{P}_{t}^{*}=\left\{\begin{array}{ll}
\underline{\mathcal{P}}_{t}^{*} & \text { for } t<0 \\
\overline{\mathcal{P}}_{t}^{*} & \text { for } t \geq 0
\end{array},\right.
$$

where $\overline{\mathcal{P}}_{t}^{*}>\underline{\mathcal{P}}_{t}^{*}$. This jump reflects a permanent shock to the exchange rate that leads to an increase in the GBP-denominated price of imported intermediates. ${ }^{22}$ As can be seen from Figures 1a and 2, this simplifying assumption of the permanent shock to $\mathcal{P}_{t}^{*}$ aligns with the depreciation of the GBP exchange in the year following the Brexit referendum.

\section{Two types of nominal rigidities}

We consider the price-setting decisions of firms in the two sectors $i=1,2$ in this model under two different forms of nominal rigidities. Both of them involve the firms spending an amount of time, $\ell_{i, j, t}$, in case they decide to adjust their prices. Because the menu cost is in terms of time, the actual cost of changing prices is given by $\ell_{i, j, t} P_{t} \mathcal{W}_{t}$.

Following Dotsey, King and Wolman (1999), this menu cost is independently drawn in each period and across producers of varieties from the distribution function $\Lambda(\ell):[0, \infty) \rightarrow[0,1]$. In the time-dependent price-setting model, based on Calvo (1983), that we consider

$$
\Lambda(\ell)=\delta \text { for } 0<\ell<\infty,
$$

such that firms can freely adjust their prices with a probability $\delta$ and otherwise adjusting prices is infinitely expensive. In the state-dependent menu cost model

$$
\Lambda(\ell)= \begin{cases}0 & \text { for } \ell<\bar{\ell} \\ 1 & \text { for } \ell \geq \bar{\ell}\end{cases}
$$

Thus, in this case, the menu cost distribution is degenerate and all firms face a certain (fixed) menu cost equal to $\bar{\ell}$.

In both cases, firms set their prices in order to maximize the expected present discounted value of their current and future profits. They do so in response to the path of the sector-

\footnotetext{
${ }^{22} \mathrm{We}$ assume this shock is permanent in order to avoid having to make specific assumptions about the stochastic process that drives $\mathcal{P}_{t}^{*}$. Our results can be interpreted as the limiting case in which this process becomes increasingly persistent in the $\overline{\mathcal{P}}_{t}^{*}$ state.
} 
specific relative price level, $\mathcal{P}_{i, t}$. Equilibrium is that path of $\mathcal{P}_{i, t}$ for which the firms' price setting decisions aggregate to this path of the sector-specific relative price level. ${ }^{23}$

\subsection{Transitional dynamics in response to a Brexit-like shock}

To compare these two models with the data, we consider the difference in impulse response of the two sectors with respect to the various components of the dynamics of the distribution of $\log$ prices that we introduced to a permanent $10 \%$ increase in the price of imports. ${ }^{24}$ This increase is in line with the depreciation of the GBP we observed in the data following the Brexit referendum.

We present the impulse responses for a set of parameters that is chosen as follows. We choose the elasticities of substitution, $\varepsilon$ and $\theta$, as well as the cost share of imports in sector $i=2, \alpha$, based on evidence from other data. In particular, consistent with the evidence from Hobijn and Nechio (2018) on the inflation response to VAT changes in the E.U., we set $\varepsilon=3$ to $\theta=1$. The cost share of imports in sector $i=2$ is $\alpha=0.4$ to match the evidence in Table 1. We set the steady-state rate of inflation, $\bar{\pi}$ to 2 percent annualized, which is the Bank of England's inflation target as of $2016 .^{25}$

The remaining parameters of the model are the persistence, $\rho$, and standard deviation, $\sigma_{u}$, of the idiosyncratic productivity shocks as well as the menu cost parameters, $\delta$ for the time-dependent and $\bar{\ell}$ for the state-dependent model. We choose them to match the model's steady-state properties in terms of the components of (3) and (5) with the summary statistics for the whole sample (All) over the benchmark period reported in Table 3. In particular, we match the average incidence of price changes, $\delta_{t}$, the variance of inflation, $\sigma_{\pi, t}^{2}$, and price disparities, $\sigma_{p, t}^{2}{ }^{26}$

The resulting impulse response functions are reported in Figures 5 and 6 for equations (3) and (5) respectively. The blue short-dashed line with square markers shows those for the menu-cost model and the red dashed line with diamond markers for the Calve model.

Figure 5 shows that both the Calvo and the menu cost models map well the dynamics of the

\footnotetext{
${ }^{23}$ The associated Bellman equations as well as the solution method we apply to solve for the transitional dynamics of these models are discussed in Appendix Section A.2.

${ }^{24}$ In terms of the model parameters this means that $\underline{\mathcal{P}}_{t}^{*}=1$ for all periods before June 2016 and $\overline{\mathcal{P}}_{t}^{*}=1.10$ for all periods after that date in equation (14).

${ }^{25}$ This choice of steady-state inflation rate yields a slightly higher than average inflation in our sample over the benchmark period (2001m1-2007m12). This does not affect the qualitative properties of our results. Small changes in the other parameters neither do as well.

${ }^{26}$ Because the steady-state inflation rates is set to $2 \%$ annualized, matching the incidence of price changes pins down the steady-state magnitude of price changes. Similarly because in the steady state, variance of log prices is constant (i.e., its change is zero), matching the variance of inflation pins down the mean reversion of inflation. The matched moments and parameter calibration are reported in the Appendix Table A6.
} 
mean log price in response to shocks. The Calvo model, however, performs better in matching the movements of the magnitude of price adjustments, as evidenced by panel (c). The menu cost model implies a speedier adjustment than the data suggests.

Figure 6 shows that that both the Calvo and the menu models are able to decently match the dynamics of the variance of $\log$ prices. However, because of the importance of the response of the mean reversion of inflation in determining the dynamics of this variance, the menu cost model fares better than the Calvo model. The bottom panels of the Figure help understand why. The data shows that the importance of the adjustment through the endogenous selection of the incidence of price changes (panel (g)), which by definition is zero in the Calvo model. Note however, that while the state dependent model captures well the response of the mean reversion of incidence, it completely misses the response of the mean reversion of the magnitude. This is so because in the data low prices adjust faster than higher ones, while inherently in a menu cost model it is the higher prices that adjust faster due to the narrowing of the SS bands.

The comparison between empirical and model-based impulse responses functions depicted in Figures 5 and 6 suggest that the data exhibits properties of both types of models. On the one hand, a time-dependent pricing model performs relatively well in replicating the dynamics of some of the first-order moments of mean log prices. In particular, that model matches particularly well the response of the magnitude of price adjustments, which corresponds to the bulk of the response of mean log prices in the data. This finding suggest that if one's interest is to estimate the effects of a shock on aggregate inflation, relying a Calvo model is appropriate, even when the shock is as large as the one we consider in our exercise. On the other hand, if one's interest is to match the response of the variance of log prices, a state dependent model would fare better since this model is able to capture the endogenous selection in the incidence of price changes, which seems to be of particular relevance in the data.

Finally, we note that the fact that we can clearly distinguish between models and match the data with a state-dependent pricing model has to do with the size of the shock we focus on. The fact that the Brexit shock is large is important because, as Klenow and Kryvtsov (2008) and Alvarez, Lippi and Passadore (2016) have argued, many of these models are almost observationally equivalent, and thus indistinguishable, under small shocks.

\section{Conclusion}

In this study, we exploit the quasi-experiment that stemmed from the depreciation of the British pound following the unexpected passing of the Brexit referendum. Using micro-level price quote data that underpins the official U.K. CPI, we develop a novel methodology to decompose the 
change in the dispersion of prices at the granular item, region, and shop level which allows us to quantify how the cost shock transmits through to the distribution of prices as well as inflation. Our results show that a large portion (about 75 percent) of the inflationary effect effect from Brexit was attributable to the magnitude of price changes, conditional on the price adjusting. Due to the importance of the magnitude-effect, we show that a time-dependent price-setting model can match the dynamics of the inflation response following the cost shock fairly well.

Our results assessing the response of the distribution of prices allow us to assess which firms adjust prices, and by how much, following a cost shock. The data show that selection effects occur in response to a cost shock. Specifically, firms at the lower end of the price distributionthat is, firms with relatively low prices - are more apt to adjust their price. Time-dependent models inherently do not allow for any selection effects, meaning these types of models will misrepresent how the distribution of prices evolves following a cost shock. The state-dependent pricing model is better able to match some of these distributional effects, as it accurately captures the selection of price changes from the lower end of the price distribution. However, the state-dependent model misses on where in the distribution magnitude of price changes is largest, predicting that high-price firms (low productivity) adjust by more conditional on changing their price, while the data suggest the opposite is true. This result in the state-dependent model is at least partly attributable to the constant-elasticity of substitution assumption, which leads to the SS bands narrowing at higher levels of productivity (see Appendix Figure A6b). It is plausible that models allowing for nonconstant elasticity of substitution and other price-setting mechanisms may alleviate this discrepancy of the model with the data. More generally, a potentially interesting avenue for future research would be to better uncover why the statedependent model misses on this front.

\section{References}

Abadie, Alberto, Alexis Diamond, and Jens Hainmueller. 2010. "Synthetic Control Methods for Comparative Case Studies: Estimating the Effect of California's Tobacco Control Program." Journal of the American Statistical Association, 105(490): 493-505.

Allington, Nigel F.B., Paul A. Kattuman, and Florian A. Waldmann. 2005. "One Market, One Money, One Price?" International Journal of Central Banking, 1(3).

Alvarez, Fernando, Francesco Lippi, and Juan Passadore. 2016. "Are State- and TimeDependent Models Really Different?" NBER Macroeconomics Annual 2016, Volume 31, 379-457. University of Chicago Press. 
Auer, Raphael A., and Raphael S. Schoenle. 2016. "Market structure and exchange rate pass-through." Journal of International Economics, 98: 60 - 77.

Auer, Raphael, Ariel Burstein, and Sarah M. Lein. 2017. "Price and Consumption Responses to Large Exchange Rate Shocks: Evidence from the 2015 Appreciation in Switzerland." mimeo.

Bai, Jushan, and Serena Ng. 2010. "Instrumental variable estimation in a data rich environment." Econometric Theory, 26(6): 1577-1606.

Berger, David, and Joseph Vavra. 2017. "Shocks vs. Responsiveness: What Drives TimeVarying Dispersion?" National Bureau of Economic Research, Inc NBER Working Papers 23143.

Berger, David, and Joseph Vavra. 2018. "Dynamics of the U.S. price distribution." European Economic Review, 103: 60 - 82.

Bils, Mark, and Peter J. Klenow. 2004. "Some Evidence on the Importance of Sticky Prices." Journal of Political Economy, 112(5): 947-985.

Born, Benjamin, Gernot Muller, Moritz Schularick, and Petr Sedlacek. 2017. "The Economic Consequences of the Brexit Vote." C.E.P.R. Discussion Papers CEPR Discussion Papers 12454.

Breinlich, Holger, Elsa Leromain, Dennis Novy, and Thomas Sampson. 2017. "The Brexit Vote, Inflation and UK Living Standards."

Calvo, Guillermo A. 1983. "Staggered prices in a utility-maximizing framework." Journal of Monetary Economics, 12(3): 383 - 398.

Cornia, Marco, Kristopher S Gerardi, and Adam Hale Shapiro. 2012. "Price dispersion over the business cycle: Evidence from the airline industry." The Journal of Industrial Economics, 60(3): 347-373.

Dhyne, Emmanuel, Luis J. Alvarez, Herve Le Bihan, Giovanni Veronese, Daniel Dias, Johannes Hoffmann, Nicole Jonker, Patrick Lunnemann, Fabio Rumler, and Jouko Vilmunen. 2006. "Price Changes in the Euro Area and the United States: Some Facts from Individual Consumer Price Data." Journal of Economic Perspectives, 20(2): 171-192. 
Dixon, Huw David, and Kun Tian. 2017. "What We can Learn About the Behaviour of Firms from the Average Monthly Frequency of Price-Changes: An Application to the UK CPI Data." Oxford Bulletin of Economics and Statistics, 79(6): 907-932.

Dotsey, Michael, Robert G. King, and Alexander L. Wolman. 1999. "State-Dependent Pricing and the General Equilibrium Dynamics of Money and Output." The Quarterly Journal of Economics, 114(2): 655-690.

Eusepi, Stefano, Bart Hobijn, and Andrea Tambalotti. 2011. "CONDI: A Cost-ofNominal-Distortions Index." American Economic Journal: Macroeconomics, 3(3): 53-91.

Fair, Ray C., and John B. Taylor. 1983. "Solution and Maximum Likelihood Estimation of Dynamic Nonlinear Rational Expectations Models." Econometrica, 51(4): 1169-1185.

Gagnon, Etienne. 2009. "Price Setting during Low and High Inflation: Evidence from Mexico." The Quarterly Journal of Economics, 124(3): 1221-1263.

Golosov, Mikhail, and Robert E. Lucas. 2007. "Menu Costs and Phillips Curves." Journal of Political Economy, 115: 171-199.

Gopinath, Gita, and Oleg Itskhoki. 2010. "Frequency of Price Adjustment and PassThrough." The Quarterly Journal of Economics, 125(2): 675-727.

Hobijn, Bart, and Fernanda Nechio. 2018. "Sticker Shocks: Using VAT Changes to Estimate Upper-Level Elasticities of Substitution." Journal of the European Economic Association, jvy009.

Hobijn, Bart, Federico Ravenna, and Andrea Tambalotti. 2006. "Menu Costs at Work: Restaurant Prices and the Introduction of the Euro." The Quarterly Journal of Economics, 121(3): 1103-1131.

International Monetary Fund. 2016. "United Kingdom." IMF Country Reports, 16/169.

Jordà, Òscar. 2005. "Estimation and Inference of Impulse Responses by Local Projections." American Economic Review, 95(1): 161-182.

Kehoe, Patrick, and Virgiliu Midrigan. 2015. "Prices are sticky after all." Journal of Monetary Economics, 75(C): 35-53.

Klenow, Peter J., and Benjamin A. Malin. 2010. "Microeconomic Evidence on PriceSetting." In Handbook of Monetary Economics. Vol. 3, , ed. Benjamin M. Friedman and Michael Woodford, Chapter 6, 231-284. Elsevier. 
Klenow, Peter J., and Oleksiy Kryvtsov. 2008. "State-Dependent or Time-Dependent Pricing: Does it Matter for Recent U.S. Inflation?" The Quarterly Journal of Economics, 123(3): 863-904.

Knotek, Edward S., and Stephen J. Terry. 2008. "Alternative methods of solving statedependent pricing models."

Kryvtsov, Oleksiy, and Nicolas Vincent. 2014. "On the Importance of Sales for Aggregate Price Flexibility."

Liegey, Paul R., Jr. 1993. "Adjusting Apparel Indexes in the Consumer Price Index for Quality Differences." Price Measurements and Their Uses, 209-226. University of Chicago Press.

Midrigan, Virgiliu. 2011. "Menu Costs, Multiproduct Firms, and Aggregate Fluctuations." Econometrica, 79(4): 1139-1180.

Nakamura, Emi, and Jón Steinsson. 2008. "Five Facts about Prices: A Reevaluation of Menu Cost Models." The Quarterly Journal of Economics, 123(4): 1415-1464.

Nakamura, Emi, and Jón Steinsson. 2010. "Monetary Non-neutrality in a Multisector Menu Cost Model." The Quarterly Journal of Economics, 125(3): 961-1013.

Nakamura, Emi, Jón Steinsson, Patrick Sun, and Daniel Villar. 2016. "The Elusive Costs of Inflation: Price Dispersion during the U.S. Great Inflation." National Bureau of Economic Research, Inc NBER Working Papers 22505.

Reenen, John Van. 2016. "Brexit's Long-Run Effects on the U.K. Economy." Brookings Papers on Economic Activity, 47(2 (Fall)): 367-383.

The Economist. 2016. "Who said Brexit was a surprise?" The Economist (Online). Last updated - 2016-06-26. 
Table 1: COICOP classifications, their import content, and tradability

\begin{tabular}{|c|c|c|c|}
\hline Coicop & Description & Import content & Tradable \\
\hline 0.0 & Total & 19.4 & - \\
\hline 1.1 & Food & 25.4 & True \\
\hline 1.2 & Non-alcoholic beverages & 24.0 & True \\
\hline 2.1 & Alcoholic beverages & 20.0 & True \\
\hline 2.2 & Tobacco & 20.0 & True \\
\hline 2.3 & Narcotics & 25.8 & True \\
\hline 3.1 & Clothing & 22.9 & True \\
\hline 3.2 & Footwear & 26.6 & True \\
\hline 4.1 & Actual rentals for households & 4.8 & False \\
\hline 4.2 & Imputed rentals for households & 4.6 & False \\
\hline 4.3 & Maintenance and repair of the dwelling & 24.6 & True \\
\hline 4.4 & Water supply and miscellaneous dwelling services & 8.7 & False \\
\hline 4.5 & Electricity, gas and other fuels & 36.5 & True \\
\hline 5.1 & Furniture, furnishings, carpets etc & 27.2 & True \\
\hline 5.2 & Household textiles & 34.6 & True \\
\hline 5.3 & Household appliances & 35.2 & True \\
\hline 5.4 & Glassware, tableware and household utensils & 32.9 & True \\
\hline 5.5 & Tools and equipment for house and garden & 34.3 & True \\
\hline 5.6 & Goods and services for household maintenance & 10.7 & False \\
\hline 6.1 & Medical products, appliances and equipment & 26.8 & True \\
\hline 6.2 & Out-patient services & 7.6 & False \\
\hline 6.3 & Hospital services & 7.6 & False \\
\hline 7.1 & Purchase of vehicles & 41.7 & True \\
\hline 7.2 & Operation of personal transport equipment & 45.3 & True \\
\hline 7.3 & Transport services & 19.3 & False \\
\hline 8.1 & Postal services & 14.4 & False \\
\hline 8.2 & Telephone and telefax equipment & 36.3 & True \\
\hline 8.3 & Telephone and telefax services & 23.2 & False \\
\hline 9.1 & Audio-visual, photo and info processing equipment & 32.4 & True \\
\hline 9.2 & Other major durables for recreation and culture & 37.2 & True \\
\hline 9.3 & Other recreational equipment etc & 24.4 & True \\
\hline 9.4 & Recreational and cultural services & 15.1 & False \\
\hline 9.5 & Newspapers, books and stationery & 18.9 & False \\
\hline 10.0 & Education & 3.6 & False \\
\hline 11.0 & Restaurants and hotels & 16.9 & False \\
\hline 12.0 & Miscellaneous goods and services & 16.8 & False \\
\hline
\end{tabular}

Note: Import content based on the 2014 Analytical Input-Output tables published by the ONS. Classification of trabability based on Allington, Kattuman and Waldmann (2005). COICOP 9.6, "package holidays", has no import content data and is omitted. 
Table 2: Observation counts for quasi-experiment sample 2015:6 to 2017:6

\begin{tabular}{r|rrr|r}
\hline & \multicolumn{3}{|c|}{ Import share tertile } & \\
Tradability classification & Low & Middle & High & Total \\
& $0-21$ & $21-34$ & $34-100$ & $0-100$ \\
\hline Nontradable (obs) & $\mathbf{3 3 1 , 1 2 2}$ & 16,956 & 44,814 & 392,892 \\
(bins) & $\mathbf{9 3 8}$ & 58 & 149 & 1,145 \\
(items) & $\mathbf{8 0}$ & 5 & 13 & 98 \\
Tradable (obs) & 341,870 & $1,034,287$ & $\mathbf{1 1 3 , 9 8 0}$ & $1,490,137$ \\
(bins) & 1,250 & 4,062 & $\mathbf{5 3 2}$ & 5,844 \\
(items) & 97 & 283 & $\mathbf{4 0}$ & 420 \\
\hline Total (obs) & 672,992 & $1,051,243$ & 158,794 & $1,883,029$ \\
(bins) & 2,188 & 4,120 & 681 & 6,989 \\
(items) & 177 & 288 & 53 & 518 \\
\hline
\end{tabular}

Note: Classification of trabability based on Allington, Kattuman and Waldmann (2005) and at a lower level of aggregation than the import content calculated from the 2014 analytical input-output tables for the U.K.. Second row of import share header is ranges of import content shares in percent included in tertiles 





Figure 1: U.K. inflation, GDP growth, and exchange rate

(a) GBP effective exchange rate, trade-weighted

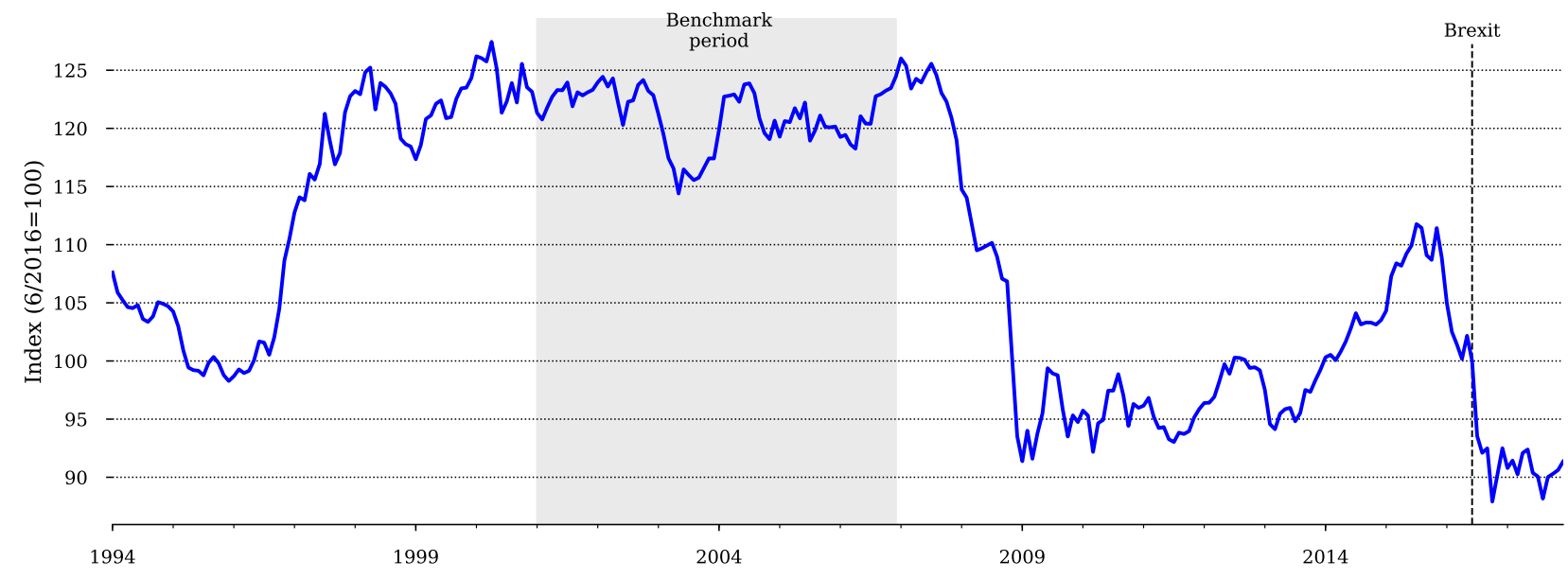

(b) GDP growth, 4-quarter growth rate

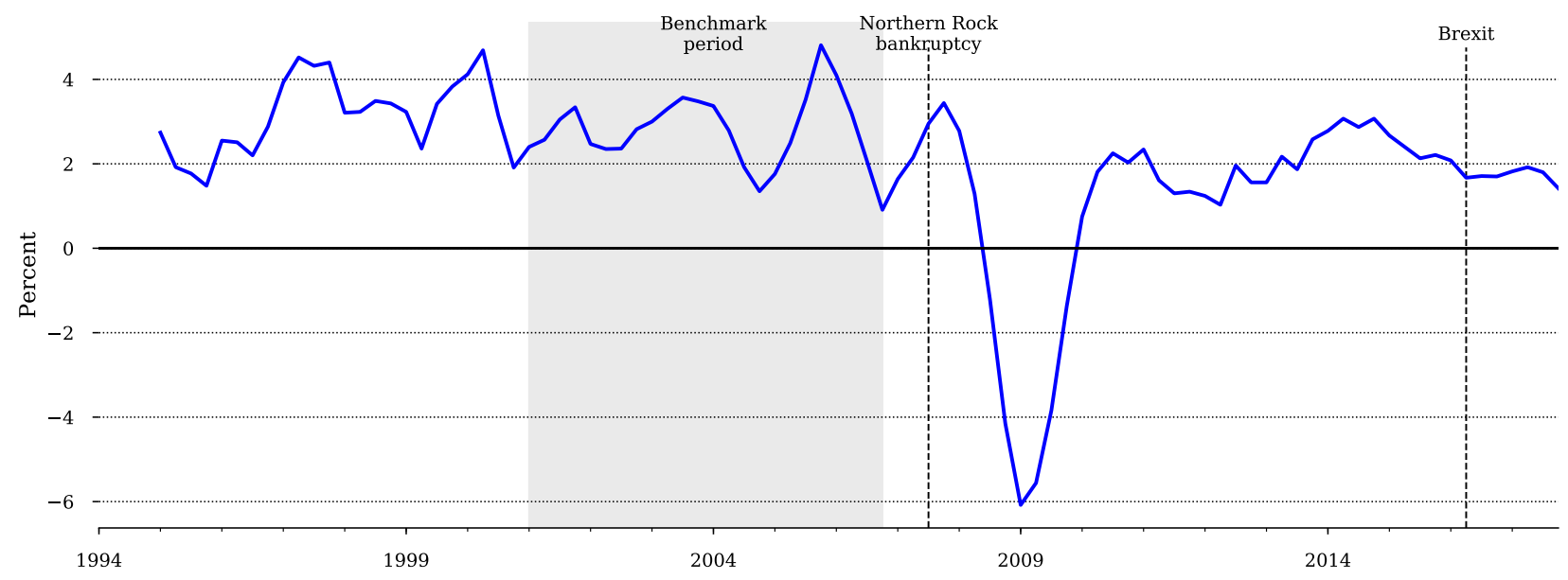

(c) CPI inflation, 12-month inflation rate

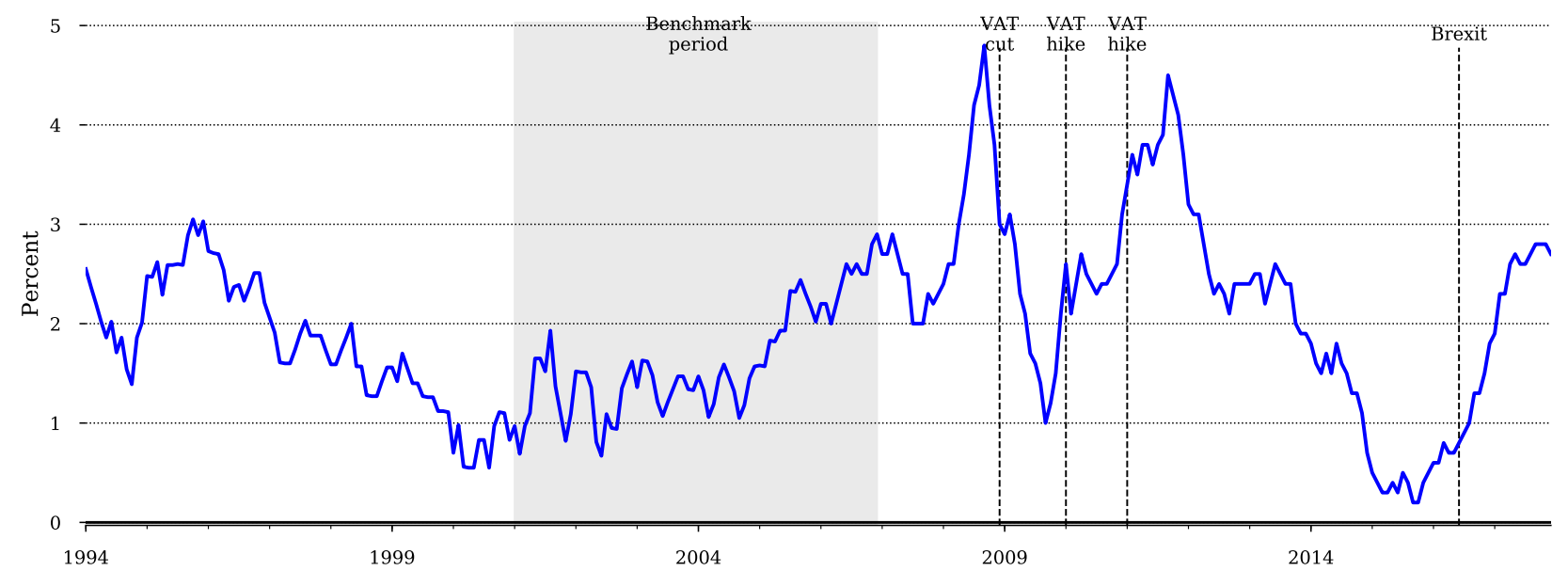

Source: (a) Bank for International Settlements (BIS), (b) ONS, (c) ONS 
Figure 2: Daily exchange rate of GBP against USD and EUR

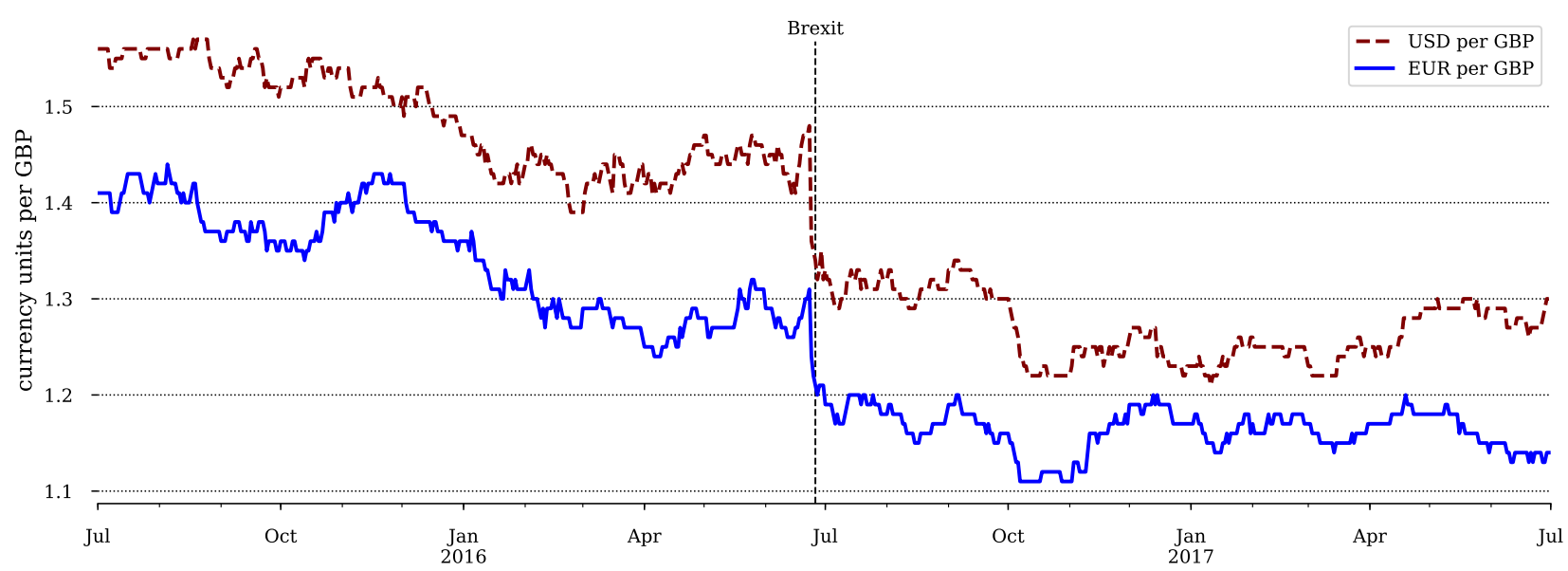

Source: Federal Reserve Board of Governors and European Central Bank

Figure 3: U.K. CPI inflation total, for tradable COICOPs, and non-tradable COICOPs

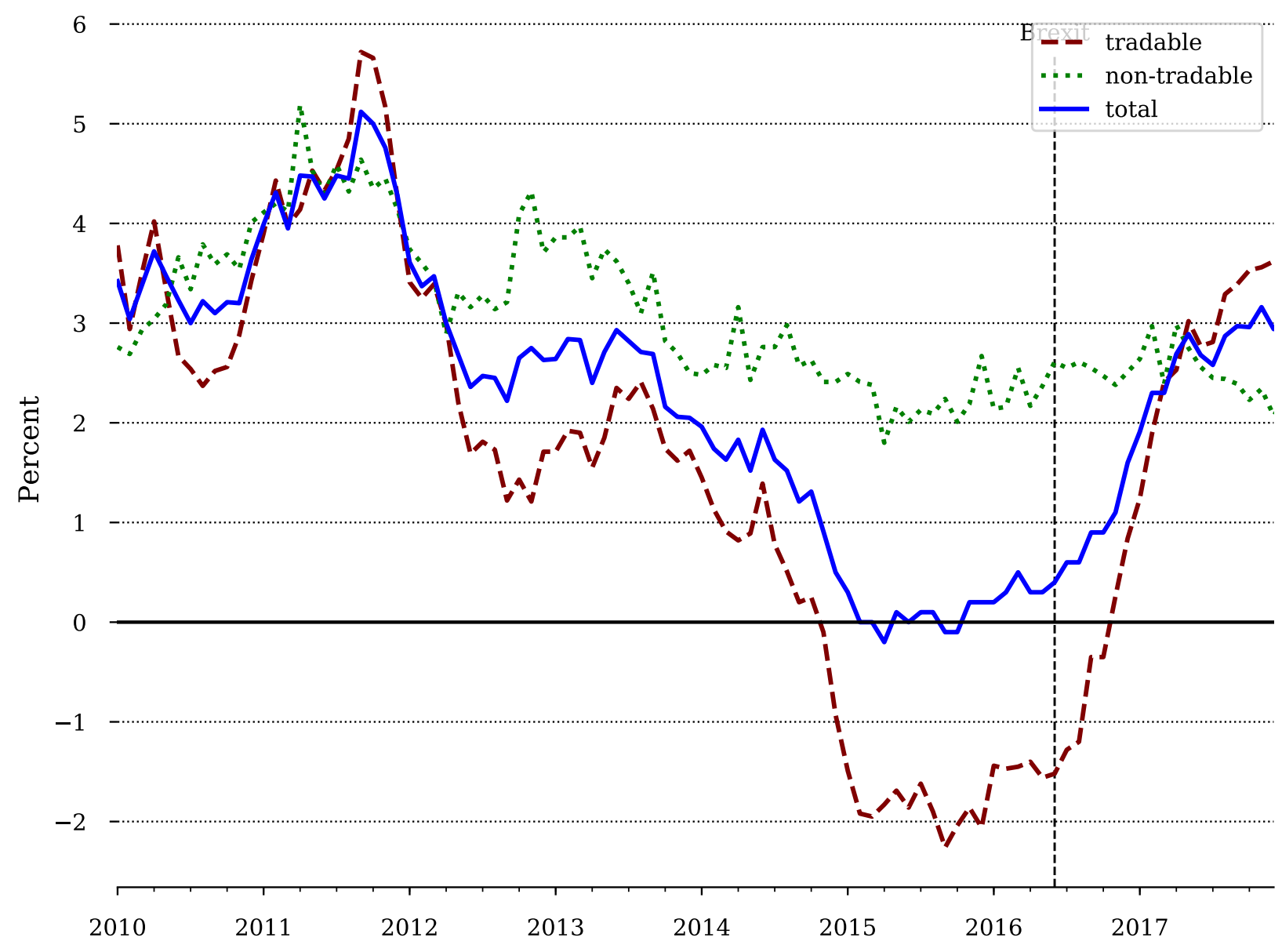

Source: ONS and authors' calculations 


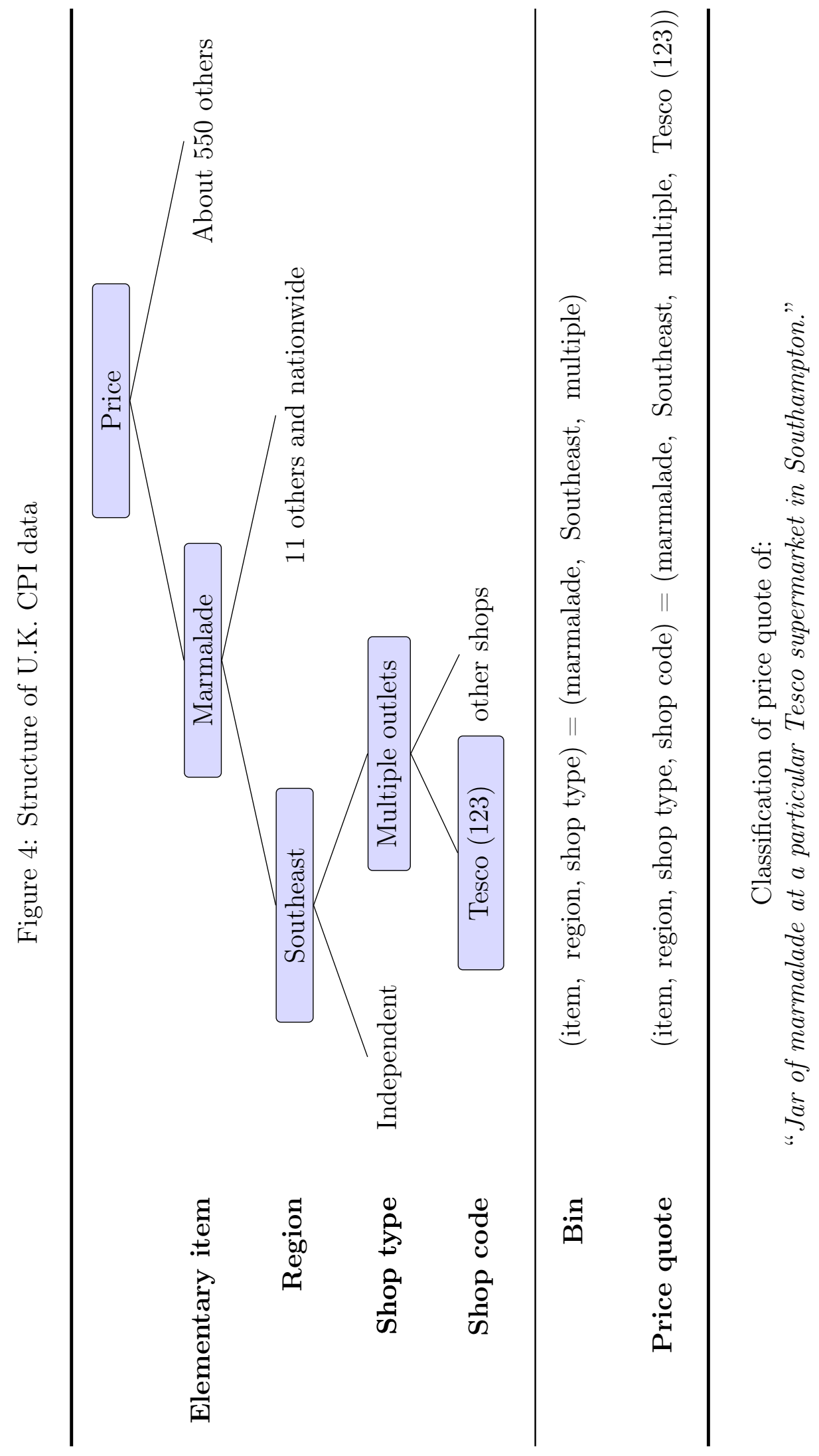


Figure 5: Model and data impulse responses: First-order moments

(a) Mean log price

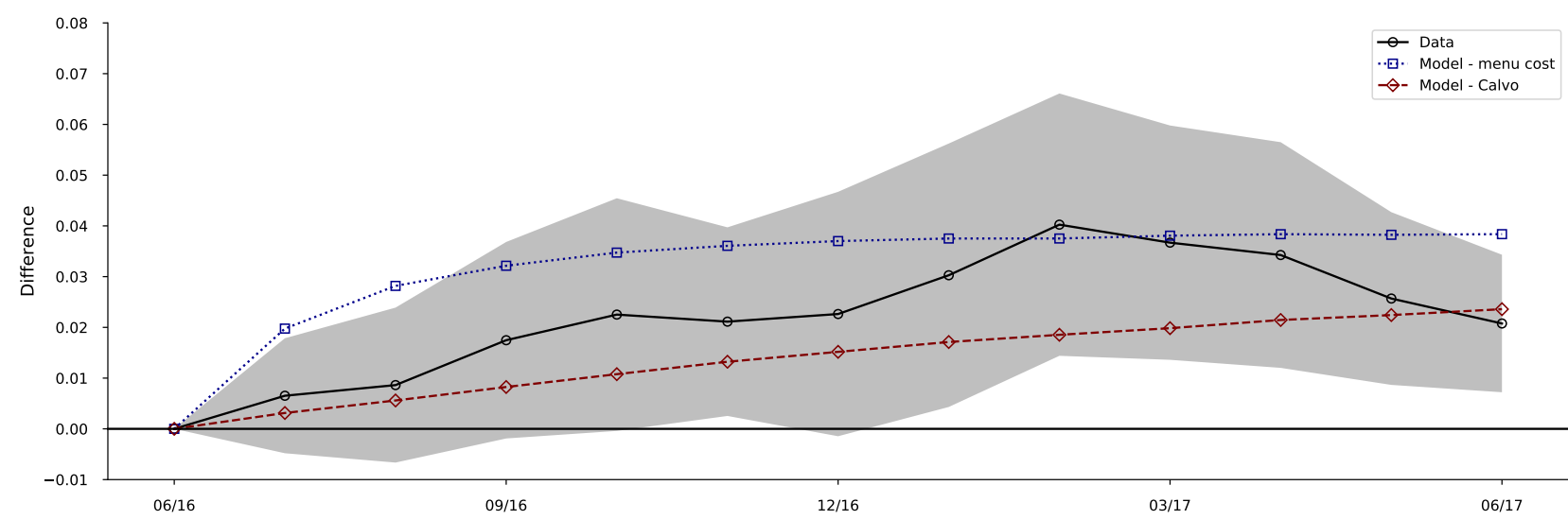

(b) Incidence

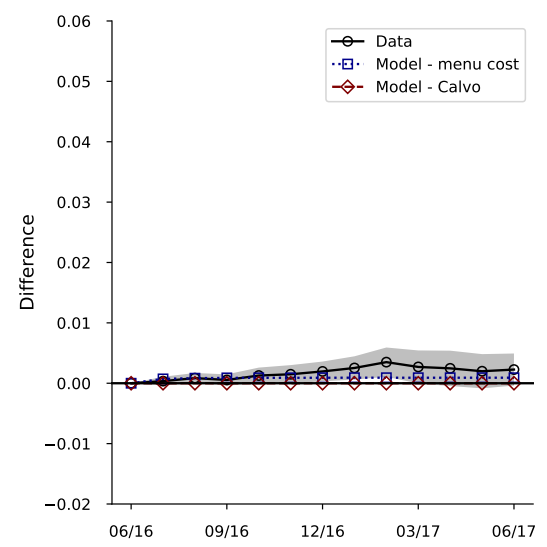

(c) Magnitude

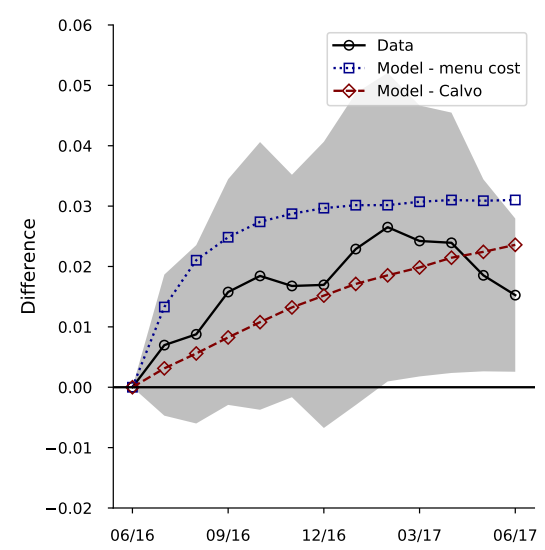

(d) Interaction

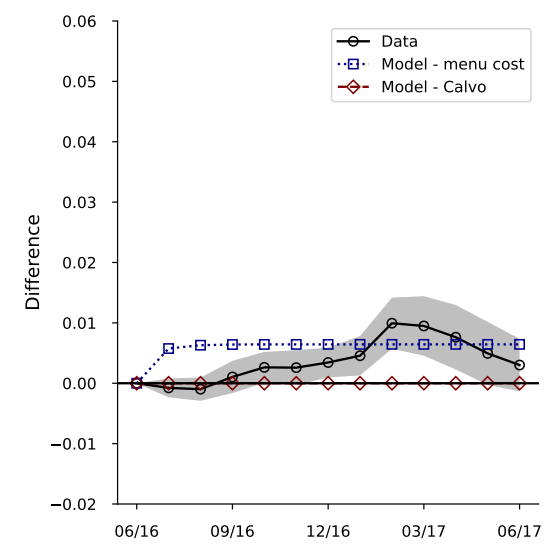


Figure 6: Model and data impulse responses: Second-order moments

(a) Variance of log prices

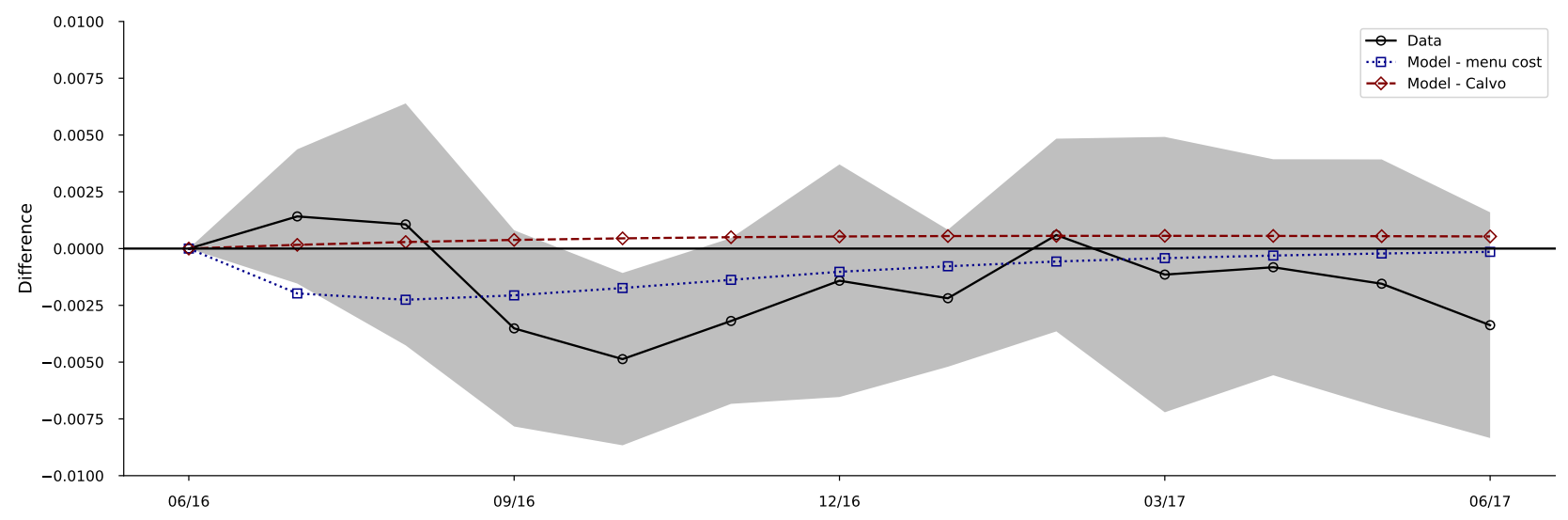

(b) Variance of inflation

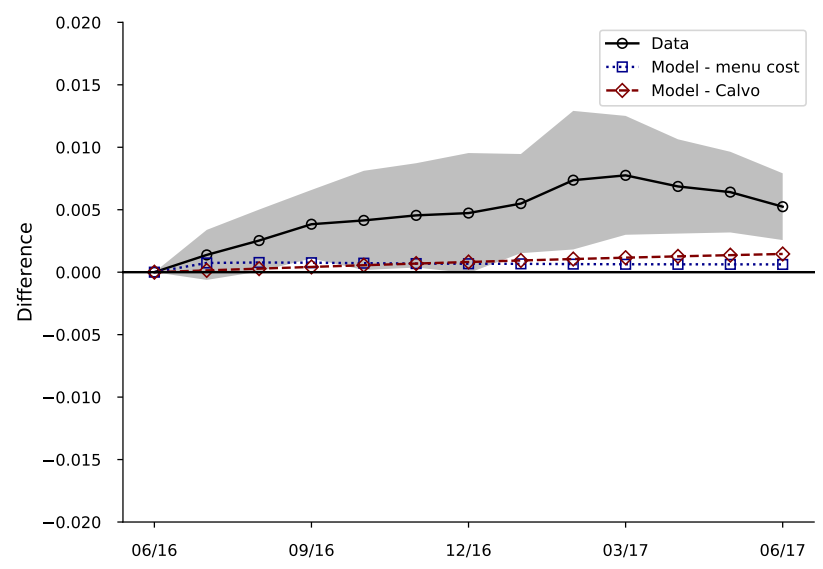

(d) Variance of magnitude

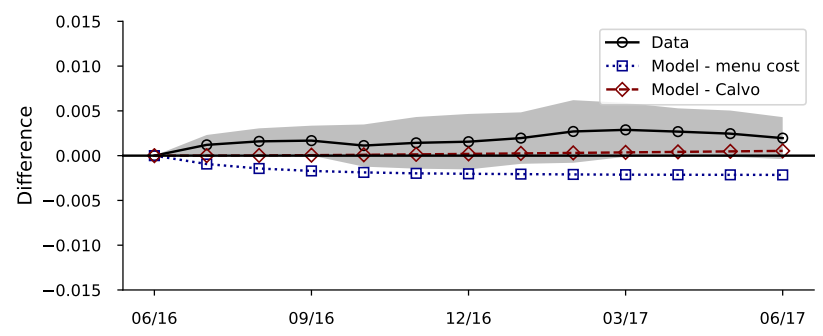

(f) Variance of incidence

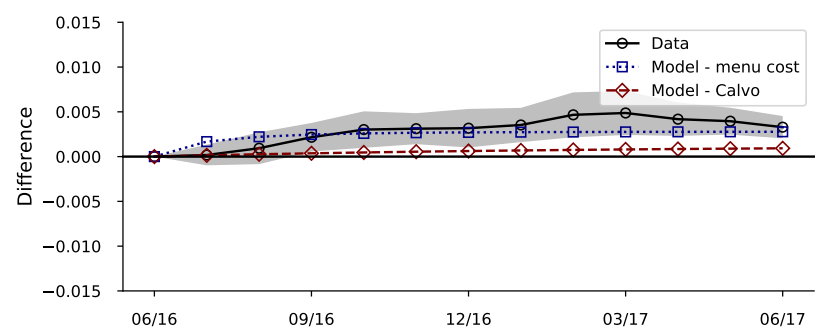

(c) Mean reversion of inflation

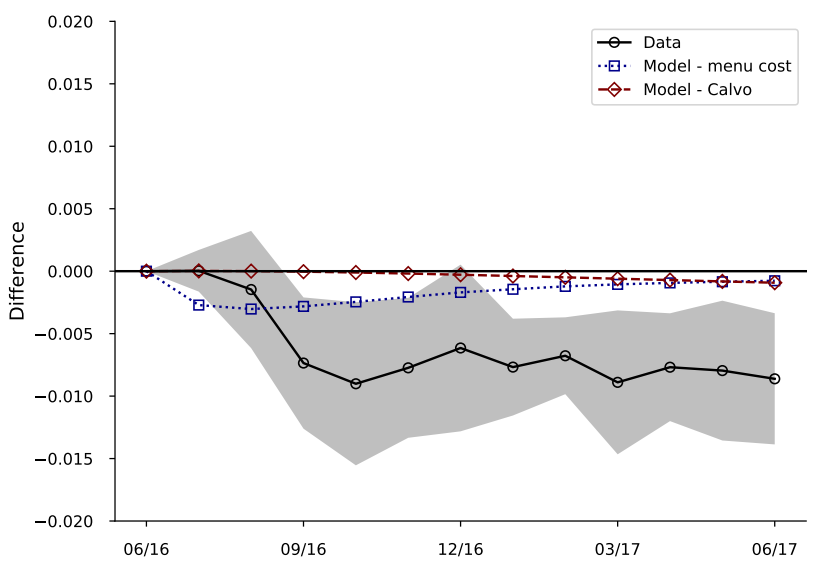

(e) Mean reversion of magnitude

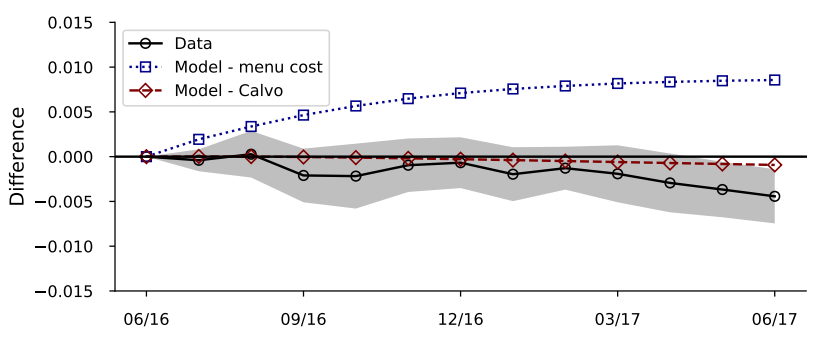

(g) Mean reversion of incidence

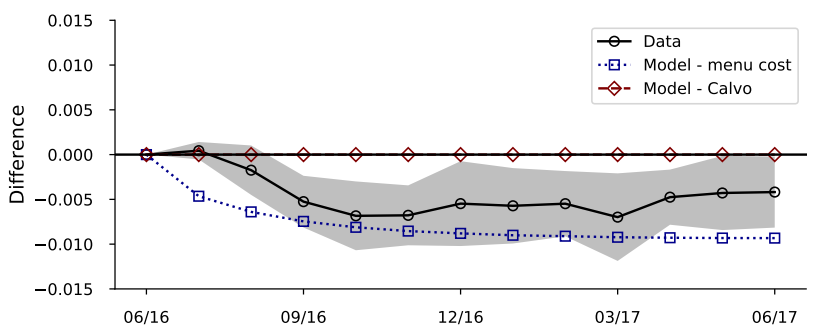




\section{Appendix:}

\section{"Using Brexit to Identify the Nature of Price Rigidities"}

\section{A Mathematical details}

\section{A.1 Details on decomposition of price dynamics}

We derive the decompositions of the change in the mean of the log prices and price disparity including entry into and exit out of the sample of price quotes. We then show how these generalized decompositions nest the those in the main text for matched models when there is no such entry and exit.

To do so, we introduce notation to capture the potential existence of non-matched price quotes in the sample of quotes in a bin in month $t$. Let the set $A$ denote all region-store-typestore-code-specific price quotes we have on an elementary item in a bin in months $t-1$ and $t$. These quotes include the matched sample, $M=S \cup C$, that we focus on in the main text. They also include two additional sets of quotes. The first are those for which we have a price quote in month $t-1$ but not in month $t$. These are the $n_{t-1}^{X}$ quotes that have exited, $E$, the sample of quotes for the bin. The second are those price quotes for which we observe the price in month $t$ but for which no price was reported in $t-1$. These are the $n_{t}^{E}$ quotes that enter the sample in month $t$. These set of all price quotes is $A=M \cup X \cup E$.

However, with entry and exit, we decompose the change in the mean and variance of the distribution of all price quotes. The set of $n_{t-1}^{A}$ price quotes in month $t-1$ is made up of the matched, $M$, and exiting, $X$, quotes, i.e., $n_{t-1}^{A}=n_{t}^{M}+n_{t-1}^{X}$. The set of price $n_{t}^{A}$ quotes in month $t$ is made up of the matched, $M$, and entering, $E$, quotes, i.e., $n_{t}^{A}=n_{t}^{M}+n_{t}^{E}$. So, what the additional notation for entry and exit allows us to do is to specifically account for sample turnover.

\section{Decomposition of the change in the sample mean of log prices}

The notation that includes entry, $E$, and exit, $X$, allows us to define the sample mean of these price quotes in months $t-1$ and $t$ as

$$
\bar{p}_{t-1}^{A}=\frac{1}{n_{t-1}^{A}} \sum_{j \in\{M, X\}} p_{j, t-1} \text { and } \bar{p}_{t}^{A}=\frac{1}{n_{t}^{A}} \sum_{j \in\{M, E\}} p_{j, t}
$$


Our focus here is on decomposing the change in this mean between months $t-1$ and $t$, i.e $\Delta \bar{p}_{t}^{A}$, in terms of the sample means conditional on changing prices, $M$, keeping prices the same, $S$, entering, $E$, and exiting, $X$.

These conditional means are defined as

$$
\bar{p}_{t-1}^{S}=\frac{1}{n_{t}^{S}} \sum_{j \in S} p_{j, t}, \bar{p}_{t-1}^{C}=\frac{1}{n_{t}^{C}} \sum_{j \in C} p_{j, t-1}, \text { and } \bar{p}_{t-1}^{X}=\frac{1}{n_{t-1}^{X}} \sum_{j \in X} p_{j, t-1}
$$

at $t-1$ and as

$$
\bar{p}_{t}^{S}=\frac{1}{n_{t}^{S}} \sum_{j \in S} p_{j, t}, \bar{p}_{t}^{C}=\frac{1}{n_{t}^{C}} \sum_{j \in C} p_{j, t}, \text { and } \bar{p}_{t}^{E}=\frac{1}{n_{t}^{E}} \sum_{j \in E} p_{j, t}
$$

at $t$. Note that, because for $j \in S$ the price does not change such that $p_{j, t}=p_{j, t-1}$, it is also the case that $\bar{p}_{t-1}^{S}=\bar{p}_{t}^{S}$.

Using these conditional means and sample sizes, we can write

$$
\bar{p}_{t-1}^{A}=\frac{n_{t}^{M}}{n_{t-1}} \bar{p}_{t-1}^{M}+\frac{n_{t-1}^{X}}{n_{t-1}} \bar{p}_{t-1}^{X},
$$

and

$$
\bar{p}_{t}^{A}=\frac{n_{t}^{M}}{n_{t}} \bar{p}_{t}^{M}+\frac{n_{t}^{E}}{n_{t}} \bar{p}_{t}^{E} .
$$

Of course, because we do not observe prices in adjacent months for the non-matched quotes in the bin, we can only calculate the inflation rate $\pi_{j, t}=p_{j, t}-p_{j, t-1}$ for matched items. The ONS uses the equally-weighted matched-model geometric price index inflation rate as the inflation rate for bin:

$$
\begin{aligned}
\pi_{t} & =\frac{1}{n_{t}^{M}} \sum_{j \in M} \Delta p_{j, t}=\bar{p}_{t}-\bar{p}_{t-1} \\
& =\left(\frac{n_{t}^{S}}{n_{t}} \bar{p}_{t}^{S}+\frac{n_{t}^{C}}{n_{t}} \bar{p}_{t}^{C}\right)-\left(\frac{n_{t}^{S}}{n_{t}} \bar{p}_{t-1}^{S}+\frac{n_{t}^{C}}{n_{t}} \bar{p}_{t-1}^{C}\right)=\frac{n_{t}^{C}}{n_{t}} \Delta \bar{p}_{t}^{C}=\delta_{t} \Delta \bar{p}_{t}^{C} .
\end{aligned}
$$

This is what we as the bin-specific inflation rate in the main text and it shows how it is the product of the incidence and magnitude of price changes in the sample of matched price quotes.

This then allows us to rewrite

$$
\begin{aligned}
\bar{p}_{t}^{A} & =\frac{n_{t}}{n_{t}^{A}} \bar{p}_{t-1}^{A}+\frac{n_{t}}{n_{t}^{A}} \pi_{t}+\frac{n_{t}^{E}}{n_{t}^{A}} \bar{p}_{t}^{E} \\
& =\frac{n_{t-1}^{A}}{n_{t}^{A}} \bar{p}_{t-1}^{A}-\frac{n_{t-1}^{X}}{n_{t}^{A}} \bar{p}_{t-1}^{X}+\frac{n_{t}}{n_{t}^{A}} \pi_{t}+\frac{n_{t}^{E}}{n_{t}^{A}} \bar{p}_{t}^{E} .
\end{aligned}
$$


In terms of the change in the conditional means of log price, this yields

$$
\Delta \bar{p}_{t}^{A}=\bar{p}_{t}^{A}-\bar{p}_{t-1}^{A}=\frac{n_{t}}{n_{t}^{A}} \pi_{t}-\frac{n_{t-1}^{X}}{n_{t}^{A}}\left(\bar{p}_{t-1}^{X}-\bar{p}_{t-1}^{A}\right)+\frac{n_{t}^{E}}{n_{t}^{A}}\left(\bar{p}_{t}^{E}-\bar{p}_{t-1}^{A}\right) .
$$

This equation captures the following intuition. The mean log price level can changes for three reasons. First, the mean of the matched price quotes changes. This is captured by the first terms that is directly related to measured inflation. Second, the mean log price of the items that drop out of the sample is not equal to that of those who remain. Third, items enter the sample at a mean log price that is different from the goods in it.

Note how when there is not entry and exit, i.e., $n_{t}^{E}=N_{t-1}^{X}=0$, the second and third terms of the above equation are zero and $n_{t}=n_{t}^{A}$. Thus, in that case, (A.10) simplifies to (A.7) used in the main text.

\section{Decomposition of the change in price dispersions}

A similar derivation can be done for the variance of log prices, i.e., price disparities. We denote this sample variance by

$$
\sigma_{A, t}^{2}=\frac{1}{n_{t}^{A}} \sum_{j \in A}\left(p_{j, t}-\bar{p}_{t}^{A}\right)^{2} .
$$

Our decomposition splits the change in this variance, $\Delta \sigma_{A, t}^{2}$, into parts related to conditional means and variances based on the four subsamples, $\{S, C, E, X\}$. In particular, we consider

$$
\begin{aligned}
\sigma_{\pi, t}^{2} & =\frac{1}{n_{t}} \sum_{j \in M}\left(\pi_{j, t}-\pi_{t}\right)^{2} \\
\sigma_{\pi p_{t-1}, t} & =\frac{1}{n_{t}} \sum_{j \in M}\left(\pi_{j, t}-\pi_{t}\right)\left(p_{j, t-1}-\bar{p}_{t-1}\right), \\
\sigma_{X, t-1}^{2} & =\frac{1}{n_{t-1}^{X}} \sum_{j \in X}\left(p_{j, t-1}-\bar{p}_{t-1}^{X}\right)^{2} \\
\sigma_{E, t}^{2} & =\frac{1}{n_{t}^{E}} \sum_{j \in E}\left(p_{j, t}-\bar{p}_{t}^{E}\right)^{2} .
\end{aligned}
$$

Given this notation, we can write

$$
\begin{aligned}
\sigma_{\pi, t}^{2} & =\frac{n_{t-1}^{A}}{n_{t}^{A}} \sigma_{A, t-1}^{2}+\frac{n_{t}}{n_{t}^{A}} \sigma_{\pi, t}^{2}+2 \frac{n_{t}}{n_{t}^{A}} \sigma_{\pi p_{t-1}, t}-\frac{n_{t-1}^{X}}{n_{t}^{A}} \sigma_{X, t-1}^{2}+\frac{n_{t}^{E}}{n_{t}^{A}} \sigma_{E, t}^{2} \\
& +\frac{n_{t}}{n_{t}^{A}}\left(\bar{p}_{t}-\bar{p}_{t}^{A}\right)^{2}-\frac{n_{t}}{n_{t}^{A}}\left(\bar{p}_{t-1}-\bar{p}_{t-1}^{A}\right)^{2}-\frac{n_{t-1}^{X}}{n_{t}^{A}}\left(\bar{p}_{t-1}^{X}-\bar{p}_{t-1}^{A}\right)^{2}+\frac{n_{t}^{E}}{n_{t}^{A}}\left(\bar{p}_{t}^{E}-\bar{p}_{t}^{A}\right)^{2}(\mathrm{~A}
\end{aligned}
$$


Writing this in terms of the first difference of the sample variance yields

$$
\begin{aligned}
\sigma_{A, t}^{2}-\sigma_{A, t-1}^{2} & =\frac{n_{t}}{n_{t}^{A}} \sigma_{\pi, t}^{2}+2 \frac{n_{t}}{n_{t}^{A}} \sigma_{\pi, p_{t-1}, t}-\frac{n_{t-1}^{X}}{n_{t}^{A}}\left(\sigma_{X, t-1}^{2}-\sigma_{A, t-1}^{2}\right)+\frac{n_{t}^{E}}{n_{t}^{A}}\left(\sigma_{E, t}^{2}-\sigma_{A, t-1}^{2}\right) A \\
& +\frac{n_{t}}{n_{t}^{A}}\left(\bar{p}_{t}-\bar{p}_{t}^{A}\right)^{2}-\frac{n_{t}}{n_{t}^{A}}\left(\bar{p}_{t-1}-\bar{p}_{t-1}^{A}\right)^{2} \\
& -\frac{n_{t-1}^{X}}{n_{t}^{A}}\left(\bar{p}_{t-1}^{X}-\bar{p}_{t-1}^{A}\right)^{2}+\frac{n_{t}^{E}}{n_{t}^{A}}\left(\bar{p}_{t}^{E}-\bar{p}_{t}^{A}\right)^{2}
\end{aligned}
$$

The interpretation of the above equation is as follows. The variance in the log prices changes for the following reasons:

- $\sigma_{\pi, t}^{2}$ : Because prices do not go up at the same rate.

- $\sigma_{\pi, p_{t-1}, t}$ : Because prices that were already high increased more than low prices (or vice versa). This is what this covariance captures.

- $\left(\sigma_{X, t-1}^{2}-\sigma_{A, t-1}^{2}\right):$ Because there was more (or less) variation in the prices of the items that exited than in the matched items.

- $\left(\sigma_{E, t}^{2}-\sigma_{A, t-1}^{2}\right)$ : Because there was more (or less) variation in the prices of the items that entered than in the matched items.

The last four terms capture the effect locational shift effects on the variance when the mean $\log$ price of exiting and entering items is different from those that are matched. Notice that, in the absence of entry and exit, i.e $n_{t}=N_{t}^{A}$ and $n_{t-1}^{X}=n_{t}^{E}=0$, all but the first two terms of the above expression are zero. These remaining two terms in the absence of entry and exit are the variance of inflation, $\sigma_{\pi, t}$, and the mean reversion of inflation, i.e., $\sigma_{\pi, p_{t-1}, t}$.

The decomposition of the change in price disparities in the main text, which abstracts from entry and exit divides the the variance of inflation, $\sigma_{\pi, t}$, up into two parts. In particular, it uses that

$$
\begin{aligned}
\sigma_{\pi, t}^{2} & =\frac{1}{n_{t}} \sum_{i}\left(\pi_{j, t}-\pi_{t}^{C}+\pi_{t}^{C}-\pi_{t}\right)^{2} \\
& =\frac{1}{n_{t}} \sum_{i}\left(\pi_{j, t}-\pi_{t}^{C}\right)^{2}+2 \frac{1}{n_{t}} \sum_{i}\left(\pi_{j, t}-\pi_{t}^{C}\right)\left(\pi_{t}^{C}-\pi_{t}\right)+\frac{1}{n_{t}} \sum_{i}\left(\bar{\pi}_{t}^{C}-\bar{\pi}_{t}\right)^{2} \\
& =\frac{n_{t}-n_{t}^{C}}{n_{t}}\left(\pi_{t}^{C}\right)^{2}+\frac{1}{n_{t}} \sum_{i \in C}\left(\pi_{j, t}-\pi_{t}^{C}\right)^{2}-\left(\pi_{t}^{C}-\pi_{t}\right)^{2} \\
& =\frac{n_{t}^{C}}{n_{t}}\left(\sigma_{\pi, t}^{C}\right)^{2}-\left(\pi_{t}^{C}-\pi_{t}\right)^{2}+\frac{n_{t}-n_{t}^{C}}{n_{t}}\left(\pi_{t}^{C}\right)^{2} \\
& =\delta_{t}\left(\sigma_{\pi, t}^{C}\right)^{2}+\left(1-\delta_{t}\right) \delta_{t}\left(\pi_{t}^{C}\right)^{2} .
\end{aligned}
$$


The first term on the last line captures that the variance of inflation across all matched items is increasing in the variance of magnitude of the changes in the price quotes in $C$. The second term captures that the variance of inflation also depends on the variance of incidence of price changes. Whether or not a matched price quote changes between months $t-1$ and $t$ can be interpreted as a draw from from a Bernoulli distribution with mean $\delta_{t}$. The associated variance is $\delta_{t}\left(1-\delta_{t}\right)$ which is what is included in the second term of the last line above.

As for the mean reversion of inflation, i.e., $\sigma_{\pi, p_{t-1}, t}$, we use that it can be split up as follows:

$$
\begin{aligned}
\sigma_{\pi, p_{t-1}, t} & =\frac{1}{n_{t}} \sum_{j \in M}\left(\pi_{j, t}-\pi_{t}\right)\left(p_{j, t-1}-\bar{p}_{t-1}\right)=\frac{1}{n_{t}} \sum_{j \in M}\left(\pi_{j, t}-\pi_{t}^{C}\right)\left(p_{j, t-1}-\bar{p}_{t-1}\right) \\
& =-\frac{n_{t}-n_{t}^{C}}{n_{t}} \pi_{t}^{C} \frac{1}{n_{t}^{S}} \sum_{j \in S}\left(p_{j, t-1}-\bar{p}_{t-1}\right)+\frac{n_{t}^{C}}{n_{t}} \frac{1}{n_{t}^{C}} \sum_{j \in C}\left(\pi_{j, t}-\pi_{t}^{C}\right)\left(p_{j, t-1}-\bar{p}_{t-1}()_{1}\right. \\
& =\delta_{t} \sigma_{\pi, p_{t-1}, t}^{C}-\left(1-\delta_{t}\right) \pi_{t}^{C}\left(\bar{p}_{t-1}^{S}-\bar{p}_{t-1}\right)=\delta_{t} \sigma_{\pi, p_{t-1}, t}^{C}+\delta_{t} \pi_{t}^{C}\left(\bar{p}_{t-1}^{C}-\bar{p}_{t-1}\right)
\end{aligned}
$$

The first term here, $\delta_{t} \sigma_{\pi, p_{t-1}, t}^{C}$, reflects the degree to which inflation reduces (or magnifies) initial differences in log prices among the prices that change. We call this the mean reversion of magnitude of price changes. The second term reflects the extent to which the fact that the prices that change are not randomly selected from the matched price quotes reduces (or magnifies) price differences. We call this the mean reversion of incidence of price changes.

Combining the above two results with equation (A.18) without entry and exit yields that

$$
\begin{aligned}
\sigma_{t}^{2}-\sigma_{t-1}^{2} & =\sigma_{\pi, t}^{2}+2 \sigma_{\pi, p_{t-1}, t} \\
& =\delta_{t}\left(\sigma_{\pi, t}^{C}\right)^{2}+\left(1-\delta_{t}\right) \delta_{t}\left(\pi_{t}^{C}\right)^{2}+2 \delta_{t} \sigma_{\pi, p_{t-1}, t}^{C}+2 \delta_{t} \pi_{t}^{C}\left(\bar{p}_{t-1}^{C}-\bar{p}_{t-1}\right)
\end{aligned}
$$

which is equation (5) in the main text.

\section{A.2 Details of model}

\section{Bellman equations and value functions}

The ex-ante, i.e., before observing the menu cost, real value function of a firm in sector $i$ with productivity level $A_{i, j, t}$ that charged the relative price $\mathcal{P}_{i, j, t-1}$ in the previous period, and faces the sectoral relative price $\mathcal{P}_{i, t}$ and the aggregate state $\mathcal{S}_{t}$, is given by:

$$
V_{i}\left(\mathcal{P}_{i, j, t-1} ; A_{i, j, t}, \mathcal{P}_{i, t}, \mathcal{S}_{t}\right)=\int_{0}^{\infty} \tilde{V}_{i}\left(\mathcal{P}_{i, j, t-1} ; A_{i, j, t}, \mathcal{P}_{i, t}, \mathcal{S}_{t} ; \ell\right) d F(\ell)
$$


The value function $\tilde{V}($.$) is the ex-post value function evaluated after the stochastic menu cost,$ $\ell_{i, j, t}$, has been realized. In particular, it is the maximum value of the two options of either keeping $(K)$ the price fixed or changing $(C)$ it net of the menu cost. That is:

$$
\tilde{V}_{i}\left(\mathcal{P}_{i, j, t-1} ; A_{i, j, t}, \mathcal{P}_{i, t}, \mathcal{S}_{t}\right)=\max \left\{V_{i}^{K}\left(\mathcal{P}_{i, j, t-1} ; A_{i, j, t}, \mathcal{P}_{i, t}, \mathcal{S}_{t}\right), V_{i}^{C}\left(A_{i, j, t}, \mathcal{P}_{i, t}, \mathcal{S}_{t}\right)\right\}
$$

where

$V_{i}^{K}\left(\mathcal{P}_{i, j, t-1}, A_{i, j, t}, \mathcal{P}_{i, t}, \mathcal{S}_{t}\right)=\Pi_{i}\left(\frac{\mathcal{P}_{i, j, t-1}}{1+\pi_{i, t}}, A_{i, j, t}, \mathcal{P}_{i, t}, \mathcal{S}_{t}\right)+\frac{1}{1+r} \mathbb{E}_{t} V_{i}\left(\frac{\mathcal{P}_{i, j, t-1}}{1+\pi_{i, t}}, A_{i, j, t+1}, \mathcal{P}_{i, t+1},, \mathcal{S}_{t+1}\right)$,

and

$V_{i}^{C}\left(\mathcal{P}_{i, j, t-1}, A_{i, j, t}, \mathcal{P}_{i, t}, \mathcal{S}_{t}\right)=\max _{\mathcal{P}_{i, j, t}}\left\{\Pi_{i}\left(\mathcal{P}_{i, j, t}, A_{i, j, t}, \mathcal{P}_{i, t}, \mathcal{S}_{t}\right)-\ell \mathcal{W}_{t}+\frac{1}{1+r} \mathbb{E}_{t} V_{i}\left(\mathcal{P}_{i, j, t}, A_{i, j, t+1}, \mathcal{P}_{i, t+1}, \mathcal{S}_{t+1}\right)\right\}$,

where $\pi_{i, t}=\frac{P_{i, t}}{P_{i, t-1}}-1$ is the inflation rate of varieties from sector $i$ and $\mathbb{E}_{t}$ denotes the expectation conditional on information at time $t$.

\section{Solution method}

The solution method that we use is a version of the Extended Path method, based on Fair and Taylor (1983). As done in Knotek and Terry (2008), we track the distribution of firms over a detailed grid over the state space $\left(A_{i, j, t}, \mathcal{P}_{i, j, t}\right)$. Because our interest is especially in the dynamics of the distribution of prices over, i.e., the marginal distribution of $\mathcal{P}_{i, j, t}$ over this state space, we use an especially fine grid along the price dimension. In particular, our grid has the dimension $(250,2500)$ and our results are very similar if we use a slightly coarser approximation.

The Extended Path solves the transitional dynamics of the model under the assumption that the economy is in steady state for $t \geq T$, where we have used $T=100$ in our solution. ${ }^{27}$ The solution method involves iterating over backwards and forwards recursions over the transitionpath until the firms' decisions aggregate to the path of the sectoral price level $\left\{\mathcal{P}_{i, t}\right\}_{t=0}^{t=T}$.

Backwards recursions For a given path of the sectoral price $\left\{\mathcal{P}_{i, t}\right\}_{t=0}^{t=T}$ this step involves solving the firms' price setting decisions. The resulting price-setting decisions do not necessarily add up to the path of the sectoral price $\left\{\mathcal{P}_{i, t}\right\}_{t=0}^{t=T}$.

Forward recursion For a given set of price-setting decisions, we update the path of the distribution of firms over the state space $\left(A_{i, j, t}, \mathcal{P}_{i, j, t}\right)$. The resulting distribution of firms over

\footnotetext{
${ }^{27}$ This choice of $T$ is so large that it does not materially affect the solution that we present.
} 
this state-space might not add up to the path of the sectoral price level $\left\{\mathcal{P}_{i, t}^{t=T}\right\}_{t=0}^{t=0}$ that their price-setting decisions are based on.

The solution method iterates over these two recursions until the path of the sectoral price level is consistent with the firms' price setting decisions.

\section{B Data Appendix}

\section{B.1 Bin construction}

Shop price quotes are collected in the United Kingdom by the Office of National Statistics (ONS) to measure inflation at a monthly rate. ONS classifies each shop price quote by: month, item, region, and shop type. A region refers to the 12 geographic regions (defined by ONS) and shop type refers to either "multiples" (chain stores with 10 or more outlets) or "independents" (less than 10 outlets). ONS defines another layer of classification, called stratification which divides the data into different "stratum." They refer to four different "stratum types:" not stratified, region, region and shop type, and shop type. They also refer to "stratum cells" which track subgroups within a stratum type. ${ }^{28}$ They provide a region identifier for all price quotes, even those with stratum type "shop type." In order to control for any regional heterogeneity across the entire data set, we added a region identifier to ONS stratum types "not stratified" and "shop type." For any given item, this places each shop quote into one of 72 "bins:"29

\begin{tabular}{ll} 
Stratum Type & \# of bins per item \\
\hline Not stratified (with region identifier) & 12 bin (1 per region) \\
Region & 12 bins (1 per region) \\
Shop type (with region identifier) & 24 bins (12 regions $\times 2$ shop types) \\
Region and Shop type & 24 bins (12 regions $\times 2$ shop types) \\
\hline
\end{tabular}

For a given region, there will be up to 6 separate bins. For example, for a given item sold in a shop in London there is a bin called "London, not stratified," "London, stratified by region," "London, multiple, stratified by shop type," "London, multiple, stratified by region/shop type," "London, independent, stratified by shop type," and "London, independent, stratified by region/shop type."

\footnotetext{
${ }^{28}$ For example, stratum type "region" has 12 cells, while stratum type "shop type" has 2 cells.

${ }^{29}$ Note that not all items are in every bin in the data.
} 


\section{B.2 Comparison of price quote data with published CPI}

The price quotes collected are aggregated up a tree of item groupings and weighting systems to eventually arrive at an overall aggregate measure of national inflation, the consumer price index (CPI). The aggregation tree from shop price quotes to headline CPI is below:

$$
\begin{gathered}
\text { shop price quotes } \rightarrow \text { item-stratum } \rightarrow \text { item } \rightarrow \text { COICOP Class } \rightarrow \text { COICOP Group } \rightarrow \\
\text { COICOP Division } \rightarrow \text { Headline }
\end{gathered}
$$

where at higher levels of aggregation, the Classification of Individual Consumption According to Purpose (COICOP) system is used. Price quotes are not available for all items in the published CPI. Specifically, price quotes are available for 61 percent of the UK data, representing 69 of the 85 COICOP classes (see columns 1 through 3 of Table A2) ${ }^{30}$ Figure A1 shows three inflation series: ${ }^{31}$ (1) the headline CPI inflation published by the ONS; (2) published item-index inflation, for all items that are available in the price quote data ${ }^{32}$; and (3) price quote inflation, using the price-quotes published by the ONS. The available price quote data does a fairly good job of representing the headline published CPI data. Furthermore, the differences between series (2) and (3) are minimal, showing the consistency of the price quote and weighting methods used in this study.

\section{B.3 Data cleaning}

We removed sales and recoveries, as well as the COICOP categories of petrol (70202) and clothing $(30102-04,30200)$. We then removed price quotes that were likely errors or that we did not have enough data per bin. Specifically, we cleaned the data with the following steps:

- Removed prices quotes ONS deemed invalid or non-comparable.

- Removed noisy bins:

- Removed all observations with less than 4 shop quotes (not frequency weighted by shop weight) in an item-bin-month.

- Dropped item-bin-months with very large confidence intervals. Construct a 95 percent confidence interval around the variance of log prices using the $\chi^{2}$ distribution,

\footnotetext{
${ }^{30}$ These 16 excluded COICOP classes are insurance connected with dwelling, insurance connected with health, insurance connected with transport, other financial services, water supply, sewage collection, electricity, gas, new cars, second hand cars, passenger transport by railway, passenger transport by air, passenger transport by sea, postal services, newspapers and books, package holidays.

${ }^{31}$ All series are year-over-year percentage change in the index.

${ }^{32}$ ONS publishes indexes aggregated up to the item level
} 
for each item-bin-month. We then drop item-bin-months with confidence intervals in the top 1 percent of all confidence intervals.

- Winsorized on the variable $\Delta \sigma_{t}^{2}$ at the item-bin level at the $1^{\text {st }}$ and $99^{\text {th }}$ percentile.

- Corrected for an error found in the price quote data where base prices were incorrectly inputed as the value .0004 . All price quotes with base price equal to .0004 are removed.

- Removed incorrect quotes from the January 1999 release. The item IDs of items removed are: 510209, 510226, 510121, 510206, 510220, 510230, 510231, 510428, 510506, 510511, $510514,510521,510523$.

- Removed other items from the January 1999 release. These item indices created from the price quote data were significantly greater than the item indices published by the ONS. We remove items in the top 15 percent of the distribution of item indices created from price quotes relative to item indices published by the ONS.

- Removed observations in which shops have the same identification code in the same region and stratum. The ONS is not authorized to release the remaining variables to uniquely identify shops.

- Dropped observations from May 2005, for which most price quote data are missing.

- Exclude price changes due to sales from the sample.

Figure A2 illustrates the effects of data cleaning on the inflation series: the time series labeled as (1) reproduces the price quote inflation series (PQ data) in Figure A1, the series labeled as (2) removes sale and recoveries, (3) removes sale and recoveries as well as clothing and petrol, and (4) reports the final cleaned data. Most of the discrepancy between the final data (series 4) and the original PQ series (series 1) stems from dropping sales. However, this is mainly a level shift as the time series patterns remain similar.

Table A2 shows the effects of data cleaning on the sample size. The final price quote data represents 54 percent of the headline CPI number, but approximately 90 percent (i.e., .54/.61) of the available price quote data.

\section{Estimation details}

We estimate equation (6) on three sets of dependent variables $\left(y_{i, t+h}\right)$ : 
(i) the log price level, $p_{i, t}$, and its three time-varying components described in equation (4):

$$
\begin{array}{r}
p_{i, t+h}-p_{i, t-1}=\beta^{h} \mathbb{B}_{t} * \mathbb{T}_{i}+\delta^{h} \mathbb{B}_{t}+\gamma_{i}+\sum_{c=1}^{3} \sum_{k=1}^{12}\left(\alpha_{c, k}^{h} \Delta C_{c, i, t-k}+\phi_{c, k}^{h} \Delta C_{c, i, t-k} * \mathbb{T}_{i}\right)+\varepsilon_{i, t}, \\
C_{c, i, t+h}-C_{c, i, t-1}=\beta^{h} \mathbb{B}_{t} * \mathbb{T}_{i}+\delta^{h} \mathbb{B}_{t}+\gamma_{i}+\sum_{c=1}^{3} \sum_{k=1}^{12}\left(\alpha_{c, k}^{h} \Delta C_{c, i, t-k}+\phi_{c, k}^{h} \Delta C_{c, i, t-k} * \mathbb{T}_{i}\right)+\varepsilon_{i, t},
\end{array}
$$

where $c=1,2,3$.

(ii) the variance of $\log$ prices, $\sigma_{i, t}$, and its components described in equation (5),

$$
\begin{array}{r}
\sigma_{i, t+h}-\sigma_{i, t-1}=\beta^{h} \mathbb{B}_{t} * \mathbb{T}_{i}+\delta^{h} \mathbb{B}_{t}+\gamma_{i}+\sum_{c=1}^{4} \sum_{k=1}^{12}\left(\alpha_{c, k}^{h} \Delta C_{c, i, t-k}+\phi_{c, k}^{h} \Delta C_{c, i, t-k} * \mathbb{T}_{i}\right)+\varepsilon_{i, t}, \\
C_{c, i, t+h}-C_{c, i, t-1}=\beta^{h} \mathbb{B}_{t} * \mathbb{T}_{i}+\delta^{h} \mathbb{B}_{t}+\gamma_{i}+\sum_{c=1}^{4} \sum_{k=1}^{12}\left(\alpha_{c, k}^{h} \Delta C_{c, i, t-k}+\phi_{c, k}^{h} \Delta C_{c, i, t-k} * \mathbb{T}_{i}\right)+\varepsilon_{i, t},
\end{array}
$$

where $c=1,2,3,4$. Note that, although we take the 4 subcomponents of equation (5) as dependent and control variables, we also consider the variance of inflation $\left(\sigma_{\pi, t}^{2}\right)$ and $\sigma_{\pi, p_{t-1}, t}$ as dependent variables.

(iii) the log price level $\left(p_{i, t+h}^{z}\right)$ of the $z \in\left\{10^{t h}, 50^{t h}, 90^{t h}\right\}$ price percentile firm

$$
p_{i, t+h}^{z}-p_{i, t-1}^{z}=\beta^{h} \mathbb{B}_{t} * \mathbb{T}_{i}+\delta^{h} \mathbb{B}_{t}+\gamma_{i}+\sum_{k=1}^{12}\left(\alpha_{k}^{h} \pi_{i, t-k}^{z}+\phi_{k}^{h} \pi_{i, t-k}^{z} * \mathbb{T}_{i}\right)+\varepsilon_{i, t},
$$

for $z=\left\{10^{\text {th }}, 50^{\text {th }}, 90^{\text {th }}\right\}$ percentiles.

For each horizon $h$ the dependent variables are constructed as such that, for the log price level we have $p_{i, t+h}-p_{i, t-1}=\sum_{\tau=1}^{h} \pi_{i, t+\tau}$. The inflation rate for item $i$ is $\pi_{i, t}=\sum \omega_{b, i} \pi_{b, i, t}$, where $\omega_{b, i}$ is the bin weight and $\pi_{b, i, t}=\sum \omega_{s, b, i} \frac{p_{s, b, i, t}}{p_{s, b, i, t-1}}$ is the matched-shop log change in prices for item $i$ in $b$ and shop $s$, and $\omega_{s, b, i}$ are shop weights provided by ONS.

For the components in equations (4) and (5), $C_{c, i, t+h}-C_{c, i, t-1}=\sum_{\tau=1}^{h} \Delta C_{c, i, t+\tau}$ for each $c \in\{1,2,3\}$ and $c \in\{1,2,3,4\}$, respectively.

Finally, $\pi_{i, t}^{z}=\sum \omega_{b, i} \pi_{b, i, t}^{z}$ and $\pi_{b, i, t}^{z}$ is the change in log price for the shop whose price was the $z^{t h}$ percentile in bin $b$ at time $t-1$ and $\omega_{b, i}$ are bin-weights provided by ONS. That is, $\pi_{b, i, t}^{z}=\left[\frac{p_{s, b, i, t}}{p_{s, b, i, t-1}} \mid p_{s, b, i, t-1}=p_{s, b, i, t-1}^{z}\right]$, where $p_{s, b, i, t-1}^{z}$ represents the $z^{\text {th }}$ percentile price (frequency weighted) in bin $b$ for item $i$. It follows that the dependent variable is the sum of the monthly 
price changes: $p_{i, t+h}^{z}-p_{i, t-1}^{z}=\sum_{\tau=1}^{h} \pi_{i, t+\tau}^{z}$. Since we do not decompose this variable, the controls include lags of the dependent variable as opposed to the components.

Results for the estimation of the regressions described in items $(i)$ and $(i i)$ are reported in the main text. Results for the estimation of the regresions described in item (iii) are reported in the Appendix Figure A3. The latter set of plots confirm the shrinking of the distribution of prices as the prices at lower percentiles are adjusted by more.

\section{C.1 Synthetic control approach}

In an ideal experiment, the treated and control units are identical, on average, in the absence of the treatment. However, the summary statistics, reported in Tables 3 and A3, indicate some differences in the treatment and control items in the nontreatment periods (i.e., the benchmark and pre-Brexit periods). Most notably, the treated group, i.e. tradables, includes items whose prices change more frequently than those in the control group. Although the local projection method used in this study includes controls aimed to correct for pre-Brexit trends, it does not account for differential effects of the treatment in the post-Brexit period. For example, flexiblypriced items may react to a cost shock differently than do sticky-priced items. Such differences in characteristics of the treated group from the comparison group could potentially convolute the empirical results.

In this section, we implement the synthetic control method of Abadie, Diamond and Hainmueller (2010) which alleviates the possible concern that the treated and control items have different characteristics. The authors propose a "synthetic" control group that can be constructed as a convex combination of control items that most closely resembles the characteristics of the treated group. They refer to this set of goods as the "donor pool," which, in our context, includes items are that are both nontradable and in the lower tertile import share. ${ }^{33}$

To construct the synthetic control group, one needs to choose the set of characteristics to be matched with the treated group. We include as predictors the frequency of price changes $\left(\delta_{t}\right)$, the inflation of price changes $\left(\pi_{t}^{C}\right)$, the variance of inflation $\left(\sigma_{\pi, t}^{2}\right)$, and the mean reversion of price changes $\left(2 \sigma_{\pi, p_{t-1}, t}\right)$. The statistics of interest are obtained by averaging over the pre-Brexit period (2015m6-2016m5). By construction, the synthetic control group closely resembles the treated group in the pre-Brexit period.

Tables A4 and A5 compare pre-Brexit mean characteristics of tradables (the treated group)

\footnotetext{
${ }^{33}$ The synthetic approach is known to be sensitive to the set of items included in the donor poll. For that reason, we exclude "books" from the donor pool, as the synthetic control method are particularly sensitive to this group of items. None of our main results of Section 5 (using local projections) change when books are excluded from that sample.
} 
and the synthetic control group. By construction, the synthetic control group closely resembles the treated group. ${ }^{34}$ Notably, Table A4 shows that the frequency of adjustment for the synthetic control and the treated group are approximately the same for almost all variables of interest.

The effect of the exchange rate shock is measured as the difference between the treated and control groups in the post Brexit period. We plot this difference in Figures A4 and A5 as a red dashed line along with the main results of our study. The results from the synthetic control method reinforce our main empirical findings. Figures A4 and A5 show that the effect of the exchange rate shocks on tradables through local projections and the synthetic control methods are quite close to one another across all variables.

\footnotetext{
${ }^{34}$ Note that the means of the treated group shown in Tables A4 and A5 are weighted (by ONS provided item weights) and therefore do not match the summary statistics in Tables 3 and A3 which are ordinary arithmetic means.
} 
Table A1: Sample set up for price quotes at bin level at times $t-1$ and $t$

Panel (a): First-order unconditional and conditional moments

\begin{tabular}{|c|c|c|c|c|c|c|c|c|c|}
\hline \multirow[b]{2}{*}{ Category } & \multirow[b]{2}{*}{ Name } & \multicolumn{2}{|c|}{ log prices } & \multicolumn{2}{|c|}{ sample size } & \multicolumn{2}{|c|}{ sample mean } & \multirow{2}{*}{$\begin{array}{c}\text { inflation } \\
t\end{array}$} & \multirow{2}{*}{$\begin{array}{c}\text { incidence } \\
t\end{array}$} \\
\hline & & $t-1$ & $t$ & $t-1$ & $t$ & $t-1$ & $t$ & & \\
\hline M & matched & $p_{j, t-1}$ & $p_{j, t}$ & $n_{t}$ & $n_{t}$ & $\bar{p}_{t-1}$ & $\bar{p}_{t}$ & $\pi_{t}=\Delta \bar{p}_{t}$ & $\delta_{t}$ \\
\hline $\mathrm{C}$ & price change & $p_{j, t-1}$ & $p_{j, t}$ & $n_{t}^{C}$ & $n_{t}^{C}$ & $\bar{p}_{t-1}^{C}$ & $\bar{p}_{t}^{C}$ & $\pi_{t}^{C}=\Delta \bar{p}_{t}^{C}$ & 1 \\
\hline S & same price & $p_{j, t}$ & $p_{j, t}$ & $n_{t}^{S}$ & $n_{t}^{S}$ & $\bar{p}_{t}^{S}$ & $\bar{p}_{t}^{S}$ & 0 & 0 \\
\hline \multicolumn{10}{|c|}{ Entry and exit of price quotes (Appendix A) } \\
\hline $\mathrm{A}$ & All & $p_{j, t-1}$ & $p_{j, t}$ & $n_{t-1}^{A}$ & $n_{t}^{A}$ & $\bar{p}_{t-1}^{A}$ & $\bar{p}_{t}^{A}$ & - & - \\
\hline $\mathrm{E}$ & entering items & - & $p_{j, t}$ & 0 & $n_{t}^{E}$ & - & $\bar{p}_{t}^{E}$ & - & - \\
\hline $\mathrm{X}$ & exiting items & $p_{j, t-1}$ & - & $n_{t-1}^{X}$ & 0 & $\bar{p}_{t-1}^{X}$ & - & - & - \\
\hline
\end{tabular}

Panel (b): Second-order unconditional and conditional moments

\begin{tabular}{|c|c|c|c|c|c|}
\hline \multirow[b]{2}{*}{ Category } & \multirow[b]{2}{*}{ Name } & \multicolumn{3}{|c|}{ sample variance } & \multirow{2}{*}{$\begin{array}{c}\text { sample covariance } \\
\text { between } \pi_{j, t} \text { and } p_{j, t-1} \\
t\end{array}$} \\
\hline & & $t-1$ & $t$ & $t$ & \\
\hline M & matched & $\sigma_{t-1}^{2}$ & $\sigma_{t}^{2}$ & $\sigma_{\pi, t}^{2}$ & $\sigma_{\pi, p_{t-1}, t-1}$ \\
\hline $\mathrm{C}$ & price change & $\sigma_{C, t-1}^{2}$ & $\sigma_{C, t}^{2}$ & $\sigma_{C, \pi, t}^{2}$ & $\sigma_{C, \pi, p_{t-1}, t-1}$ \\
\hline S & same price & $\sigma_{S, t}^{2}$ & $\sigma_{S, t}^{2}$ & 0 & 0 \\
\hline \multicolumn{6}{|c|}{ Entry and exit of price quotes (Appendix A) } \\
\hline $\mathrm{A}$ & All & $\sigma_{A, t-1}^{2}$ & $\sigma_{A, t}^{2}$ & $\sigma_{A, \pi, t}^{2}$ & - \\
\hline $\mathrm{E}$ & entering items & - & $\sigma_{E, t}^{2}$ & - & - \\
\hline $\mathrm{X}$ & exiting items & $\sigma_{X, t-1}^{2}$ & - & - & - \\
\hline
\end{tabular}

Note: For example, $\bar{p}_{t-1}^{X}=\frac{1}{n_{t-1}^{X}} \sum_{j \in X} p_{j, t-1}, \delta_{t}=\frac{n_{t}^{C}}{n_{t}^{M}} \sigma_{S, t}^{2}=\frac{1}{n_{t}^{S}} \sum_{j \in S}\left(p_{j, t}-\bar{p}_{t}^{S}\right)^{2}$, $\sigma_{C, \pi, t}^{2}=\frac{1}{n_{t}^{C}} \sum_{j \in C}\left(\pi_{j, t}-\pi_{t}^{C}\right)^{2}$, and $\sigma_{\pi, p_{t-1}, t}=\frac{1}{n_{t}} \sum_{j \in M}\left(\pi_{j, t}-\pi_{t}\right)\left(p_{j, t-1}-\bar{p}_{t-1}\right)$.

Table A2: Effects of Data Cleaning on Sample Size

\begin{tabular}{lcc|ccc}
\hline & \multicolumn{2}{c|}{ Published Aggregated Data } & \multicolumn{3}{c}{ Price Quote (PQ) Data } \\
\cline { 2 - 5 } & CPI & $\begin{array}{c}\text { Item Indexes } \\
\text { in PQ data }\end{array}$ & All & No Sales, Petrol, \\
or Clothing & Final \\
\hline Weighted Share & 1 & 0.61 & 0.61 & 0.53 & 0.52 \\
Num. Coicop Class & 85 & 69 & 69 & 64 & 64 \\
Num. Item & 1446 & 1163 & 1163 & 968 & 941 \\
Num. Bins & - & - & $2,322,346$ & $1,816,291$ & $1,599,894$ \\
Num. Obs & - & - & $26,629,004$ & $19,523,777$ & $15,190,383$ \\
\hline
\end{tabular}




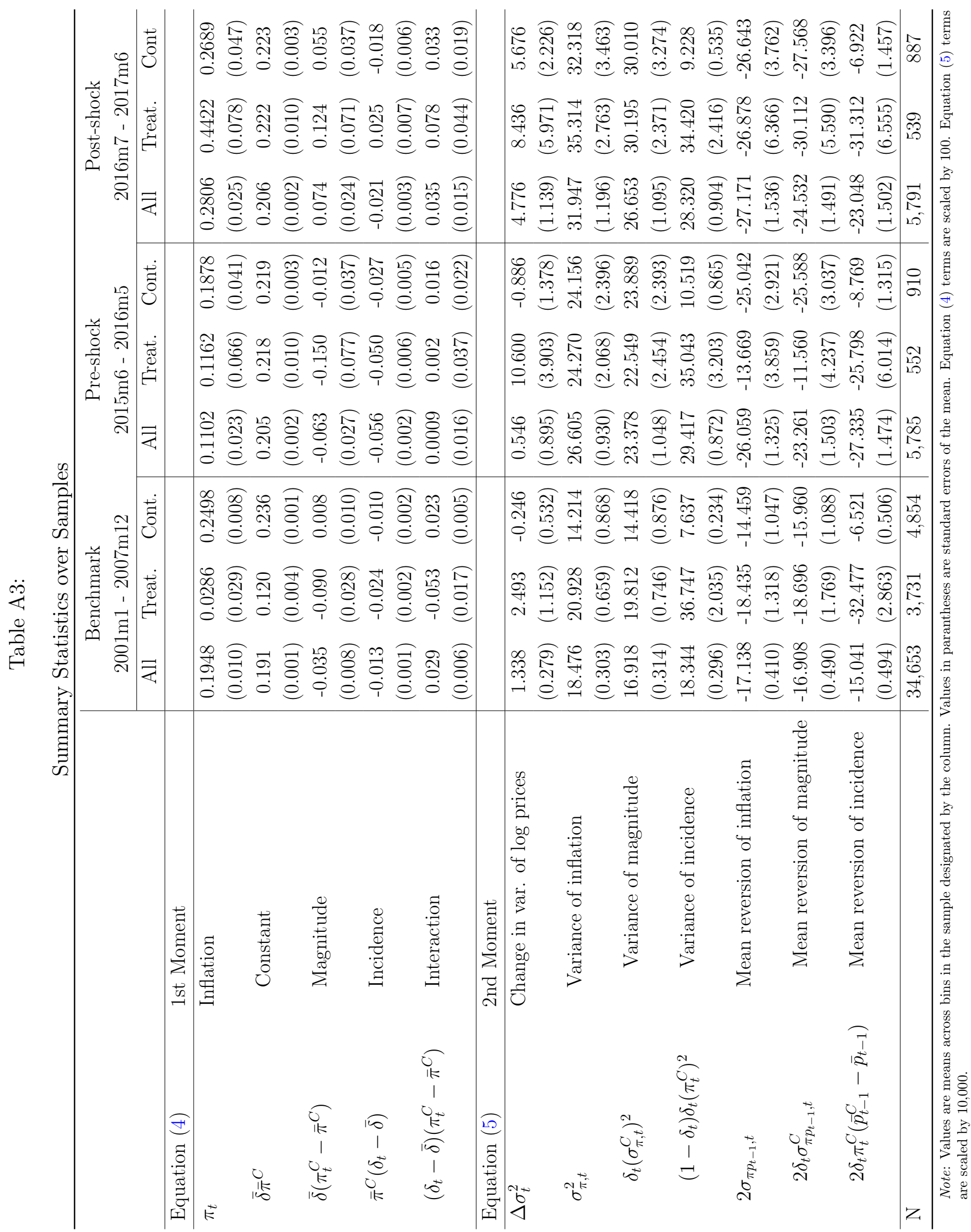


Figure A1: Published UK CPI Inflation vs. Inflation based on available price quotes

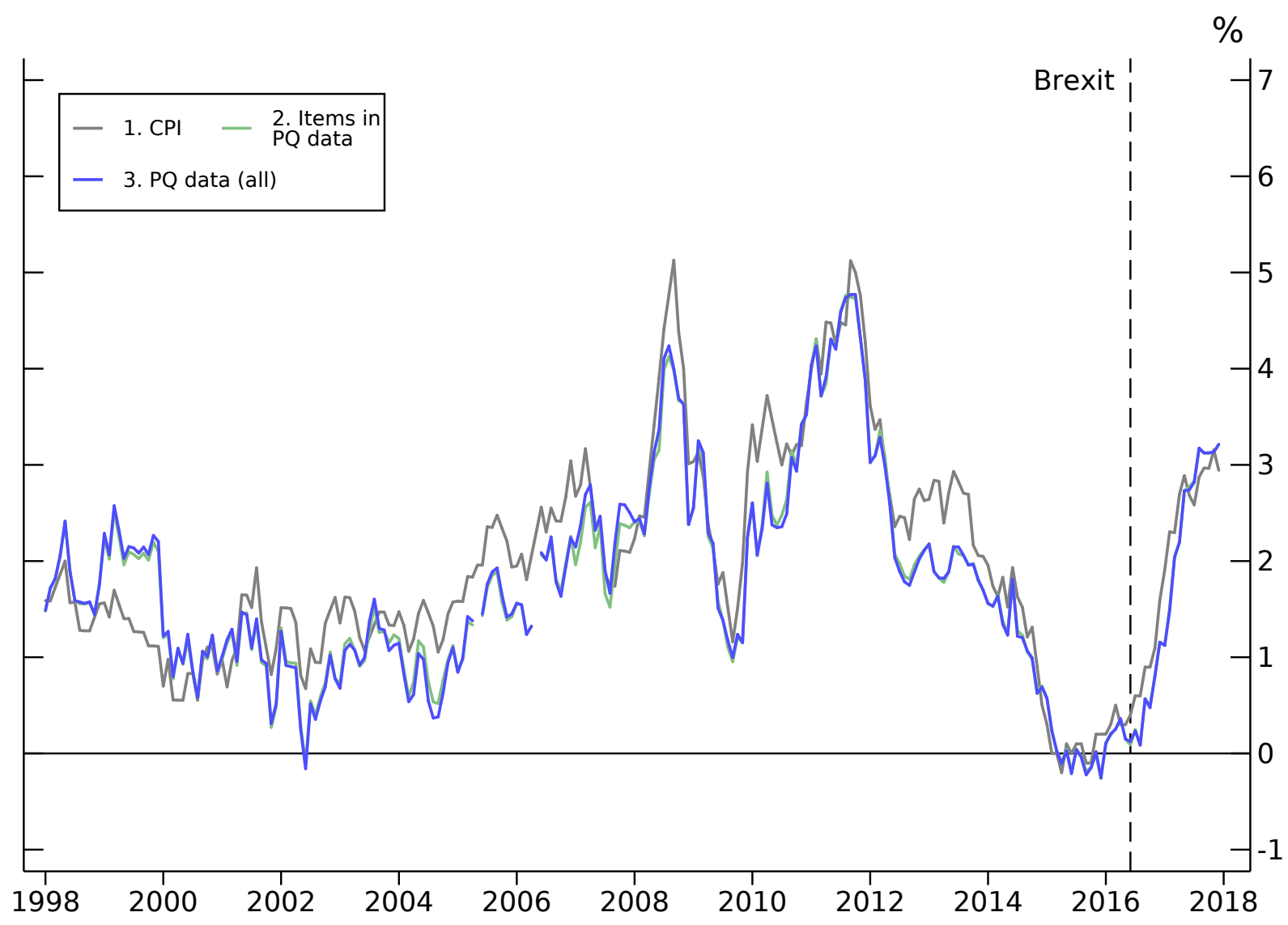

Source: ONS and authors' calculations

Note: Series (1) is the published UK CPI inflation series. Series (2) is the inflation series for all items available in the price quote data, generated using the published item indexes. Series (3) is the inflation series generated from aggregating up the price quote data. All series are year-over-year percentage changes. 
Figure A2: Effects of Data Cleaning on Price Quote Inflation

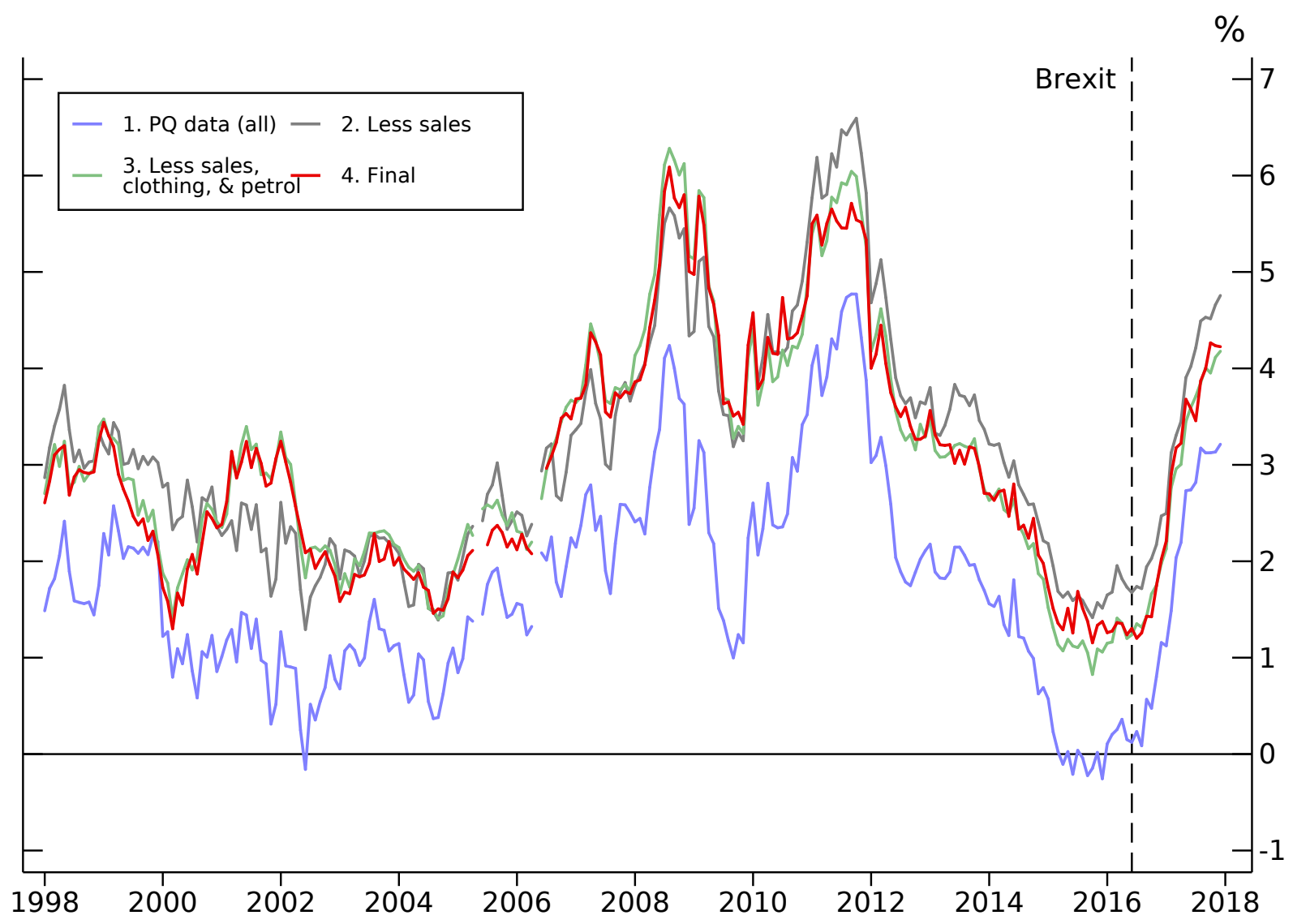

Source: ONS and authors' calculations

Note: Series (1) is the inflation series generated from aggregating up the price quote data (i.e., series (3) in Figure A1). Series (2) removes sales/recoveries. Series (3) removes sales/recoveries, petrol, and clothing. Series (4) is the final data set.

Figure A3: Brexit treatment effect on percentile of price quotes (at beginning of month) in bin.
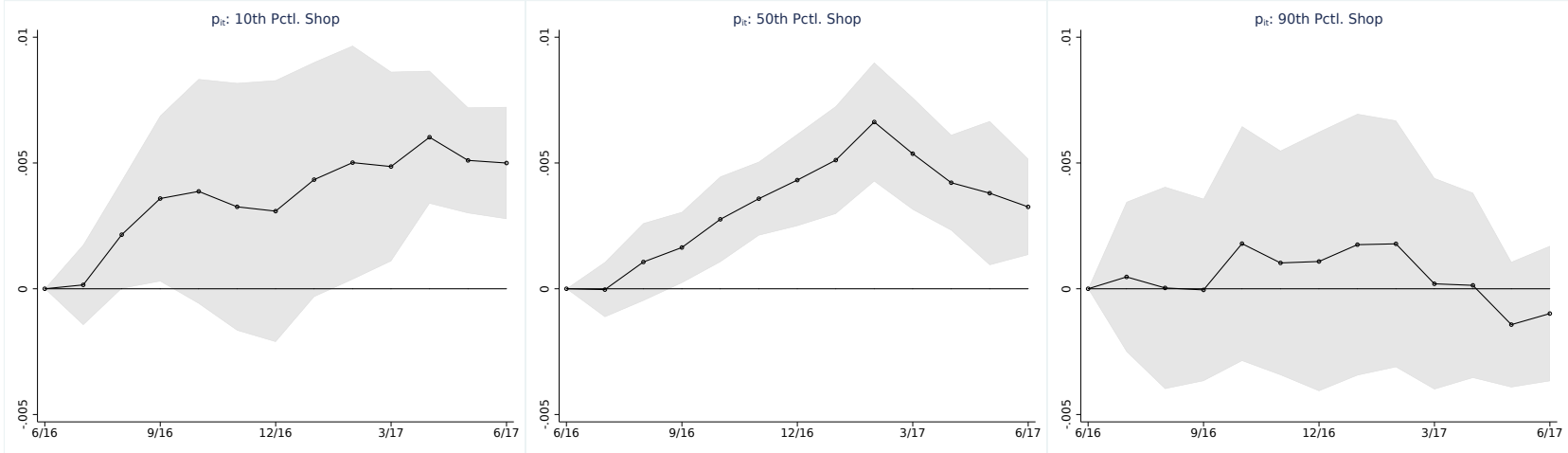

Source: ONS and authors' calculations

Note: Treatment effect in terms of average log price of $x^{\text {th }}$ percentile. Plotted are 90 percent confidence bands. 
Figure A4: Treated group minus synthetic control, first moment variables
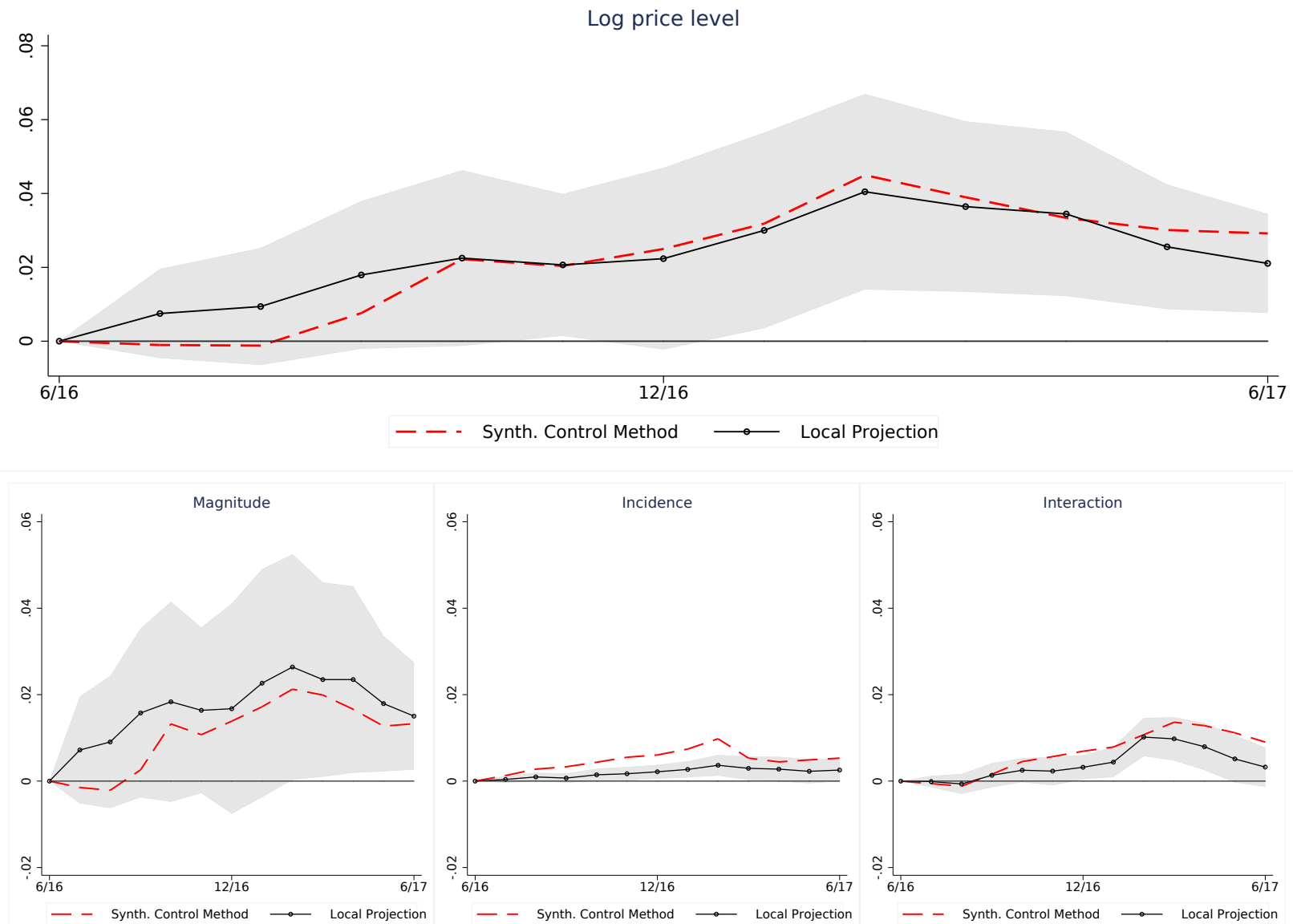
Figure A5: Treated group minus synthetic control, second moment
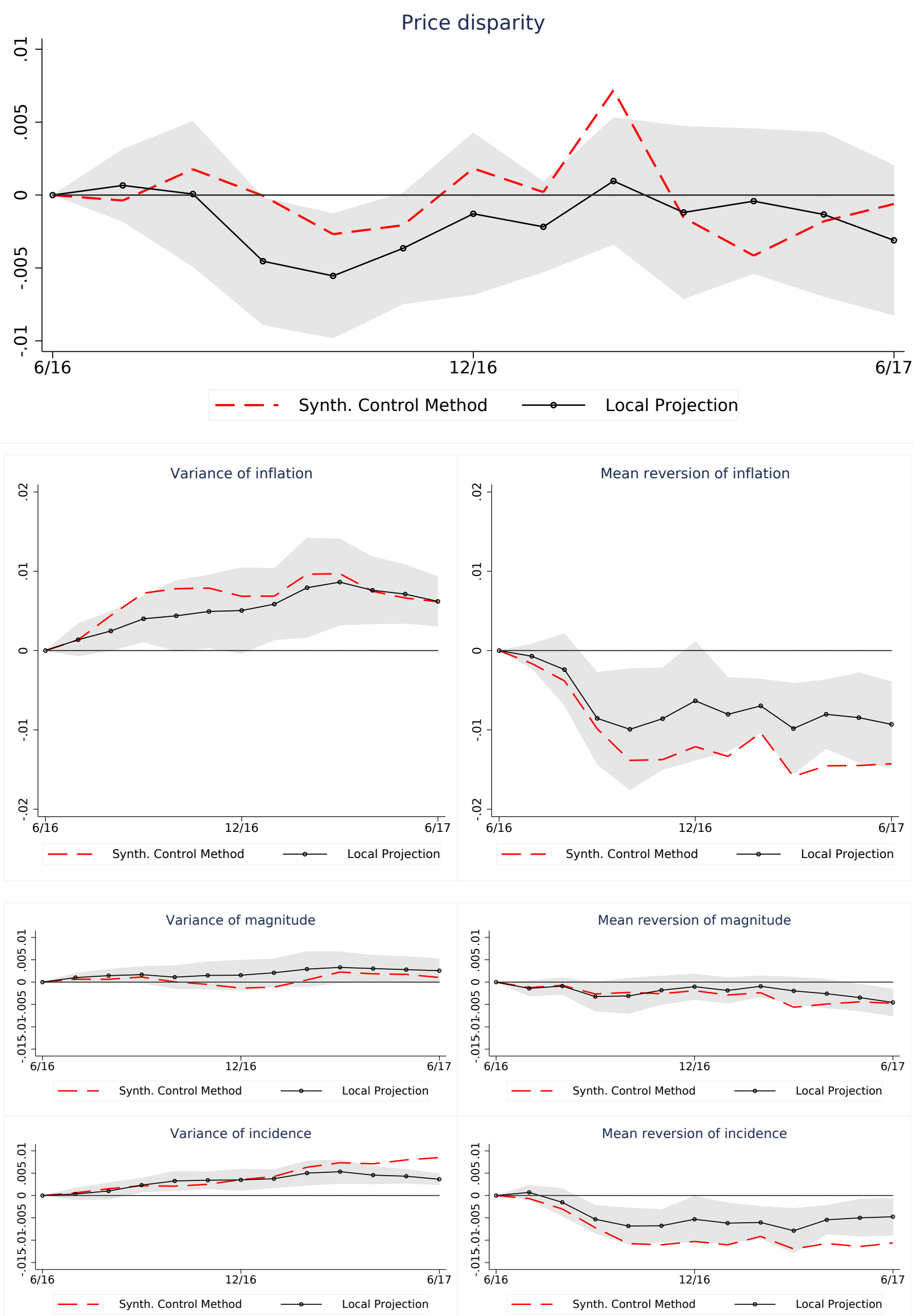
Table A6: Steady-state calibration of model parameters

\begin{tabular}{lcccc}
\hline Description & Parameter & Data & \multicolumn{2}{c}{ Model } \\
& & & Menu cost Calvo \\
\cline { 2 - 4 } & (i) Literature- and data-based parameters & & \\
Elasticity of substitution across varieties & $\varepsilon$ & - & 3 & 3 \\
Elasticity of substitution across sectors & $\theta$ & - & 1 & 1 \\
Cost-share of imports in sector 2 & $\alpha$ & - & 0.4 & 0.4
\end{tabular}

(ii) Calibrated parameters

$\begin{array}{lllll}\text { Persistence of idiosyncratic productivity shock } & \rho & - & 0.99 & 0.9825\end{array}$

Standard deviation of idiosyncratic productivity shock $\quad \sigma_{u} \quad-\quad-0.045 \quad 0.0625$

Menu cost

$\begin{array}{ll}\sigma_{u} & - \\ \ell & 0.025\end{array}$

Probability of changing prices

(iii) First and second moments of inflation and log prices

\begin{tabular}{lcccc} 
Inflation & $\bar{\pi}$ & 0.194 & 0.167 & 0.167 \\
Incidence & $\bar{\delta}$ & 0.107 & 0.108 & 0.110 \\
Magnitude & $\Delta \bar{p}^{C}$ & 2.3344 & 1.546 & 1.515 \\
Change in the variance of log prices & $\Delta \sigma^{2}$ & 0.015 & 0.000 & 0.000 \\
Variance of inflation & $\sigma_{\pi}^{2}$ & 0.184 & 0.194 & 0.203 \\
Mean reversion of inflation & $2 \sigma_{\pi, p}$ & -0.195 & -0.239 & -0.204 \\
Variance of log prices & $\sigma_{p}^{2}$ & 0.122 & 0.108 & 0.118 \\
\hline
\end{tabular}

The first two parameters on item $(i)$ are set according to the literature, while the third matches the data described on Table 2. Parameters on item (ii) are chosen such that the models nearly matches the gray shaded row parameters on item (iii). The "Data" column corresponds to the "Benchmark" column from Table 3. Steady-state inflation, $\bar{\pi}$, is calibrated to 2 percent annualized, which is slightly higher than average inflation in our sample over the benchmark period (2001m1-2007m12). 
Figure A6: Steady-state distribution of log prices and price-setting rules

(a) Distribution of log prices

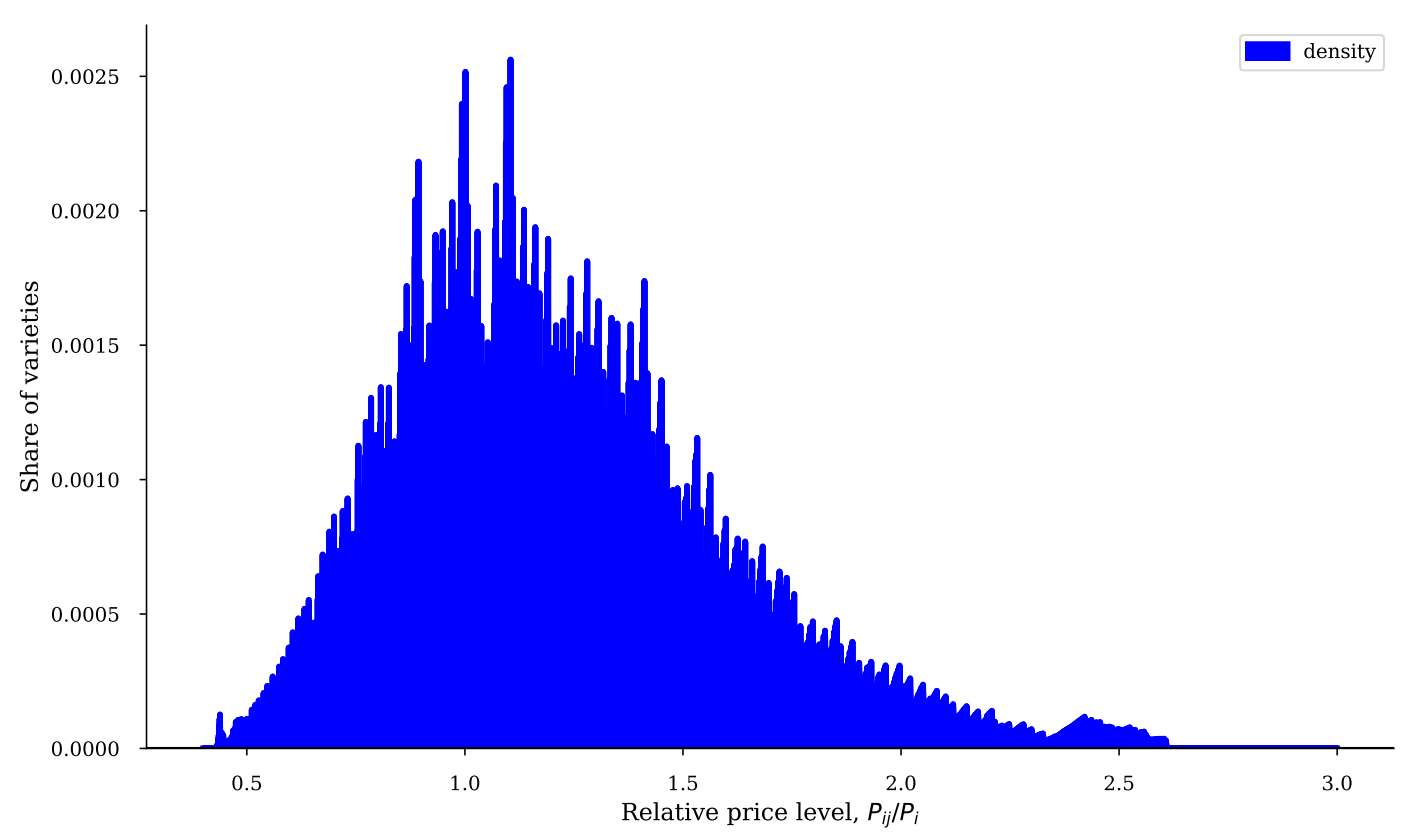

(b) Price-setting rule

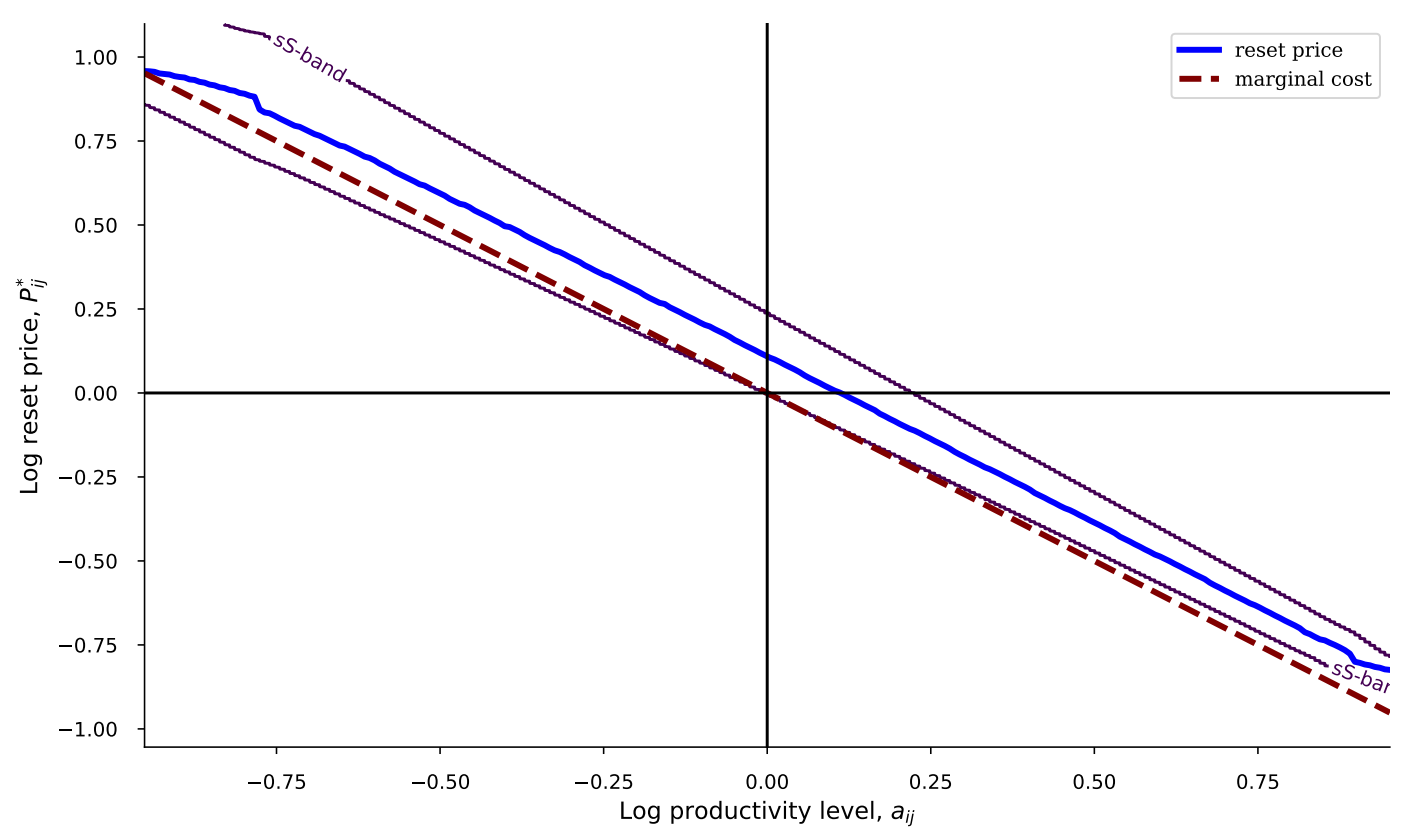

